\title{
Neurochemical models of near-death experiences: A large-scale study based on the semantic similarity of written reports
}

\author{
Charlotte Martial $^{\mathrm{a}, 1}$, Héléna Cassol ${ }^{\mathrm{a}, 1}$, Vanessa Charland-Verville ${ }^{\mathrm{a}}$, \\ Carla Pallavicini ${ }^{\mathrm{b}, \mathrm{c}}$, Camila Sanz ${ }^{\mathrm{d}}$, Federico Zamberlan ${ }^{\mathrm{b}, \mathrm{d}}$, Rocío Martínez Vivot ${ }^{\mathrm{b}, \mathrm{e}}$, \\ Fire Erowid ${ }^{\mathrm{f}}$, Earth Erowid ${ }^{\mathrm{f}}$, Steven Laureys ${ }^{\mathrm{a}}$, Bruce Greyson ${ }^{\mathrm{g}}$, Enzo Tagliazucchi ${ }^{\mathrm{b}, \mathrm{d}, \mathrm{h}, *}$ \\ ${ }^{a}$ Coma Science Group, GIGA-Consciousness and Neurology Department, University and University Hospital of Liège, Liège, Belgium \\ ${ }^{\mathrm{b}}$ Buenos Aires Physics Institute (IFIBA) and National Scientific and Technical Research Council (CONICET), Pabellón I, Ciudad Universitaria (1428), \\ Buenos Aires, Argentina. \\ ${ }^{\mathrm{c}}$ Fundación para la lucha contra las enfermedades neurológicas de la infancia (FLENI), Buenos Aires, Argentina \\ ${ }^{\mathrm{d}}$ Departamento de Física, Universidad de Buenos Aires, Buenos Aires, Argentina \\ e Instituto de Investigaciones Biomédicas (BIOMED) and National Scientific and Technical Research Council (CONICET), Buenos Aires, Argentina \\ ${ }^{\mathrm{f}}$ Erowid Center, Grass Valley, CA, United States \\ ${ }^{g}$ Department of Psychiatry and Neurobehavioral Sciences, University of Virginia Health System, United States \\ ${ }^{\mathrm{h}}$ UMR7225 Institut du cerveau et de la moelle épinière (ICM), Paris, France
}

\section{A R T I C L E I N F O}

\section{Keywords:}

Near-death experience

Psychedelics

Dissociatives

Phenomenology

\begin{abstract}
A B S T R A C T
The real or perceived proximity to death often results in a non-ordinary state of consciousness characterized by phenomenological features such as the perception of leaving the body boundaries, feelings of peace, bliss and timelessness, life review, the sensation of traveling through a tunnel and an irreversible threshold. Near-death experiences (NDEs) are comparable among individuals of different cultures, suggesting an underlying neurobiological mechanism. Anecdotal accounts of the similarity between NDEs and certain drug-induced altered states of consciousness prompted us to perform a large-scale comparative analysis of these experiences. After assessing the semantic similarity between $\approx 15,000$ reports linked to the use of 165 psychoactive substances and $625 \mathrm{NDE}$ narratives, we determined that the N-methyl-D-aspartate (NMDA) receptor antagonist ketamine consistently resulted in reports most similar to those associated with NDEs. Ketamine was followed by Salvia divinorum (a plant containing a potent and selective $\kappa$ receptor agonist) and a series of serotonergic psychedelics, including the endogenous serotonin $2 \mathrm{~A}$ receptor agonist N,N-Dimethyltryptamine (DMT). This similarity was driven by semantic concepts related to consciousness of the self and the environment, but also by those associated with the therapeutic, ceremonial and religious aspects of drug use. Our analysis sheds light on the longstanding link between certain drugs and the experience of "dying", suggests that ketamine could be used as a safe and reversible experimental model for NDE phenomenology, and supports the speculation that endogenous NMDA antagonists with neuroprotective properties may be released in the proximity of death.
\end{abstract}

\footnotetext{
* Corresponding author at: Buenos Aires Physics Institute (IFIBA) and National Scientific and Technical Research Council (CONICET), Buenos Aires, Argentina.

E-mail address: tagliazucchi.enzo@googlemail.com (E. Tagliazucchi).

${ }^{1}$ Equal contribution.
} 


\section{Introduction}

"And one or two people have said to me, and I've said it to myself: That's what death is going to be like. And, oh, what fun it will be!" Gerald Heard on the psychedelic experience.

Throughout known history, humans have shown a tendency towards modifying their ordinary state of consciousness by consuming certain fungi and plant materials (Devereux, 1997; Escohotado, 1999). Naturally occurring molecules that interact with endogenous neurotransmitters and neuromodulators can induce an ample range of effects, such as sedation, stimulation, euphoria, modifications in the perception of the environment and the self, and complex and interactive hallucinations (Lewin \& Wirth, 1931; Nichols, 2016; Perrine, 1996). From Paleolithic cave art to contemporary music the ineffability of drug-induced altered states of consciousness led to artistic expressions attempting to communicate the associated subjective experiences (Devereux, 1997; Shapiro, 1988). Frequently, analogies are made between the experiences elicited by certain psychoactive compounds and other non-ordinary states of consciousness (e.g. dreams, nightmares, sleep paralysis, mystical experiences, meditative states) (Barrett \& Griffiths, 2018; Kraehenmann, 2017; Luke, 2008; Millière, Carhart-Harris, Roseman, Trautwein, \& Berkovich-Ohana, 2018; Sanz, Zamberlan, Erowid, Erowid, \& Tagliazucchi, 2018). Certain psychiatric conditions have been compared ("modeled") to the acute effects of psychoactive compounds (Bubeníková-Valesová, Horácek, Vrajová, \& Höschl, 2008; Kyzar, Nichols, Gainetdinov, Nichols, \& Kalueff, 2017; Nichols, 2016). Such comparisons are especially common for drugs collectively known as hallucinogens, a class that includes serotonergic psychedelics (the most known example being lysergic acid diethylamide [LSD]), dissociatives (e.g. arylcyclohexalamines such as ketamine and phencyclidine [PCP]) and deliriants (e.g. Solanaceae plants rich in anticholinergic tropane alkaloids). It is also documented that dopaminergic stimulants such as methamphetamine can induce psychotic hallucinatory states (McKetin, 2018).

Perhaps the most intriguing of all experiences compared to drug-induced altered states of consciousness is that of "death" or "dying" (Grof \& Halifax, 1977; Leary, Metzner, Ram, \& Karma glin, 1966; Strassman, 2001). Such comparison is evident in the etymology of word "ayahuasca" ("vine of the dead" in the Quechua language), an Amazonian concoction with psychedelic effects induced by N,N-Dimethyltryptamine (DMT) (Schultes \& Raffauf, 2004; Schultes, Hofmann, \& Rätsch, 2001). It is documented that the Tukano and Shipibo people believe that ayahuasca allows a transition towards a state of direct communication with the spirits of the deceased (Luna, 2011). Certain societies indigenous to North America incorporate peyote (Lophophora williamsii, a cactus containing the psychedelic molecule mescaline) in their ceremonies to allow a glimpse into their conception of an afterlife (Bailey, 1973). The Bwiti people native to present-day Gabon employ the bark of Iboga (which contains the potent hallucinogenic tryptamine ibogaine) in combination with polyrhythmic music to induce "near-death" trances (Maas \& Süster, 2003). In contemporary Western society, the experiences elicited by natural and semi-synthetic hallucinogens have also been compared to dying, e.g. see the above reproduced quotation by Gerald Heard, when discussing the LSD experience with psychiatrist Sidney Cohen (Lee \& Shlain, 1992). The feeling of death and rebirth attributed to certain psychedelics has been postulated as a component of their potential therapeutic effect (Grof \& Halifax, 1977). Descriptions of dissociative anesthetics, especially ketamine, abound in comparisons with the experience of dying (Corazza \& Schifano, 2010; Jansen, 1990, 1997a,1997b). Timothy Leary, a key figure in the popularization and subsequent stigmatization of LSD, referred to ketamine experiences as "experiments in voluntary death" (Jansen, 1997a,1997b). It must be noted that the comparison between the experiences induced by these drugs and dying occurs at a purely psychological level, since these substances are very safe under most circumstances (Alberich et al., 2017; Albott et al., 2018; Dos Santos, Bouso, Alcázar-Córcoles, \& Hallak, 2018; dos Santos, 2013; Gasser et al., 2014; Glue et al., 2018; Kyzar et al., 2017; Nichols, 2016; Rabi, 2016; Riva-Posse et al., 2018).

"Dying" as a non-ordinary state of consciousness can be related to events known as near-death experiences (NDEs), i.e. phenomenological experiences associated with real or perceived proximity to death (Moody, 1976, Greyson, 2008, Sleutjes, MoreiraAlmeida, \& Greyson, 2014). The concept and terminology of NDEs were first introduced into the English language by Raymond Moody over four decades ago (Moody, 1975). The French equivalent "expériences de mort imminente" had already been identified by the French epistemologist Victor Egger decades before. The first descriptions of NDEs included a set of phenomenological features that were subsequently refined, ordered and quantified by numerical scales (Moody, 1975, Ring, 1982, Greyson, 1983). These features include some of the following: leaving the body boundaries (either towards a different space or dimension, or to observe the body from a third-person perspective, including phenomena such as autoscopy and out-of-body experiences [OBEs]), feelings of peace and bliss, traveling through a tunnel or void towards a light, sensing the presence of an irreversible threshold, review of past life events, and communicating with sentient and autonomous entities (Moody, 1975, Owens, Cook, \& Stevenson, 1990, Greyson, 1993, Kelly, 2001, Greyson, 2008, Katz, Saadon-Grosman, \& Arzy, 2017, Moore \& Greyson, 2017, Cassol et al., 2018). While some authors proposed a certain preferred temporal ordering for these events, recent research failed to detect prototypical sequences in a large sample of NDEs (Martial et al., 2017). Interestingly, similar phenomenological experiences, termed "NDE-like", have been reported in situations where there was no genuine threat to the individuals' life (e.g. after fainting or a period of anxiety (Facco \& Agrillo, 2012, Charland-Verville et al., 2014)). These experiences raise questions about the extent to which the proximity to death is involved in the appearance of a subjective NDE phenomenology. In addition, many researchers have independently noticed the phenomenological overlap between NDEs and the experiences induced by serotonergic and dissociative psychedelics (Jansen, 1997a,1997b, 1989, 1990; Strassman, 2001; Timmermann et al., 2018). Thus, it has been hypothesized that the long-standing association between these psychoactive compounds and the experience of "dying" could be based on their capacity to emulate NDEs.

The association between drug-induced experiences and NDEs is frequently discussed in terms of anecdotal evidence and firstperson accounts. Direct experimental evidence supporting this association is scarce. Corazza et al. retrospectively compared reports of 36 subjects who experienced NDEs (related to cardiac arrest) with the experiences of 36 subjects under the acute effects of ketamine 
(Corazza \& Schifano, 2010). The authors found sufficient commonalities to conclude that ketamine can reproduce at least some aspects of NDE phenomenology. Recently, Timmermann and colleagues performed a within-subjects placebo-controlled study with 13 participants to test the phenomenological similarities between the DMT state and NDEs (Timmermann et al., 2018). They found significant differences vs. placebo using numerical scales designed to quantify NDE (Greyson, 1983). Furthermore, the authors detected a significant overlap between the phenomenological features of DMT-induced experiences and those described by a matched group who reported a NDE after a life-threatening situation.

The aforementioned studies provide unique and valuable evidence, but also limitations related to the use of structured questionnaires to quantify and compare highly complex and heterogeneous experiences, as well as biases in the selection of the psychoactive compounds to be contrasted with NDEs. Indeed, NDE phenomenology has also been compared to that induced by LSD (Morse, Venecia, \& Milstein, 1989), dextromethorphan (DXM) (Luke, 2008), carbon dioxide (Meduna, 1950), hashish (Siegel, 1984), 5-MeO-DMT (the 5-methoxylated analogue of DMT) (Shulgin \& Shulgin, 1997), ayahuasca (orally ingested DMT, enabled by its combination with $\beta$-carbolines present in Baanisteriopsis caapi) (Strassman, 2001), ibogaine (Maas \& Süster, 2003), and others. Thus, the following questions remain open: (1) Is the phenomenological similarity between NDEs and drug-induced experiences highest for substances within a certain pharmacological class and mechanism of action?; (2) Can this similarity be inferred from unstructured free narratives, and do they offer insights complementary to the use of structured questionnaires?; (3) Given the heterogeneity in the events associated with NDEs (e.g. surrounding circumstances, proximity to death, emotional valence attributed by the subject, etc.), does such heterogeneity reflect on the similarity with the phenomenology of drug-induced states of consciousness?

In the present work we seek to provide an answer to these questions by retrospectively comparing 625 narratives of events classified as NDEs with a large number of reports ( $>15,000)$ spanning experiences with 165 psychoactive substances of ten different pharmacological classes. We build upon previous work showing that natural language processing (NLP) tools used to obtain the semantic similarity between narratives provide an unstructured alternative to quantify the phenomenological similarity of different reported experiences (Lange, Greyson, \& Houran, 2015; Sanz et al., 2018). Finally, we discuss how our results shed light on the historical association between certain psychoactive compounds and the experience of "dying", on the possible neurobiological mechanisms underlying the robust and reproducible phenomenology of NDEs, on the possibility that NDEs are associated with the release of endogenous neuroprotective agents in the proximity of death, and finally on the possible therapeutic uses of pharmacologically inducing NDEs in the terminally ill.

\section{Materials and methods}

In the following, we describe the corpus of NDE narratives ("NDE corpus"), the demographics of the experiencers and the known circumstances at the time of the NDEs, as well as the corpus of free narratives of experiences with psychoactive compounds obtained from the Erowid Experience Vaults ("Erowid corpus"). We present an overview of the NLP tools applied to the corpora, especially of latent semantic analysis (LSA) (Landauer, Laham, \& Derr, 2004), the core method to quantitatively compare the similarity of the reported experiences.

\subsection{NDE corpus}

NDE experiencers were recruited via the Division of Perceptual Studies (University of Virginia Health System, USA) and the GIGAConsciousness (Coma Science Group, University of Liège and University Hospital of Liège, Belgium). Completion of the anonymous questionnaire was voluntary. The NDE corpus consisted of 625 retrospective narratives of NDEs, comprising a total of 623.926 words. All experiences scored 7 or higher on the NDE Scale (15.2 \pm 7), except for 182 accounts which were collected before the development of such scale (Greyson, 1983). In those cases, the narratives contained enough detail to suggest they would have scored 7 or higher if the experiencers had completed the scale. Many narratives included additional information concerning participant demographics, nature of the circumstances surrounding loss of consciousness during the experience, severity of physical trauma/closeness to death, and self-assessment of the emotional valence of the experience (using a 3-point Likert scale ranging from -1 pleasant, 0 neutral, to +1 unpleasant). The age of the participants ( $68 \%$ females) at the time of the experience was $31 \pm 15$ years $(7 \mathrm{missing}$ values), and the age at the time of the study was $52 \pm 13$ years ( 2 missing values). Loss of consciousness at time of the NDE was attributed to head injury in $13 \%$ of the cases, to anesthesia/drug use in $30 \%$ of the cases, and to cardiac arrest in $10 \%$ of the cases (228 missing values). Severity of physical trauma/closeness to death was rated retrospectively by the experiencers as "not serious" in $27 \%$ of the cases, as "serious" in $37 \%$ of the cases and as involving loss of vital signs in 35\% of the cases (209 missing values). The NDEs were rated as "pleasant" in $82 \%$ of cases, as "neutral" in $8 \%$ of cases and as "unpleasant" in $9 \%$ of cases (279 missing values). The NDE corpus metadata can be visualized in Fig. 1.

\subsection{Erowid corpus}

Reports of psychoactive substance use were downloaded from the Erowid Experience Vaults (https://erowid.org/experiences/). The webpage www.erowid.org is defined as a "member-supported organization providing access to reliable, non-judgmental information about psychoactive plants, chemicals, and related issues". Among other resources, it contains a large number ( $>20,000)$ of curated freely expressed reports associated with the use of different psychoactive substances.

As described in a previous publication (Sanz et al., 2018), we curated the Erowid corpus by performing the following steps: (1) discarded reports that resulted from the combination of different substances; (2) discarding duplicate reports appearing under more 

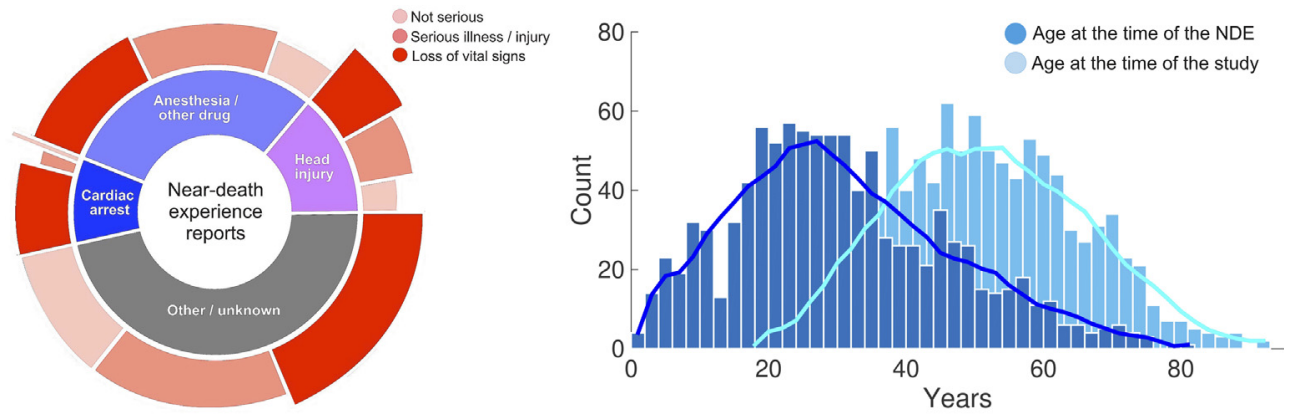

Fig. 1. Characterization of the NDE corpus metadata. Left: hierarchical pie chart showing the proportion of NDEs related to loss of consciousness due to head injury, anesthesia/drug use, cardiac arrest, and unknown causes. Each segment of the pie chart is further subdivided according to the severity of the physical trauma/closeness to death, divided into three categories: not serious, serious illness or injury, and loss of vital signs. The width of these outer segments is proportional to the average NDE Scale score (Greyson, 1983) of the experiences. Right: Histograms of the age of the experiencers at the time of the NDE, and at the time their accounts were retrospectively recollected.

than one category; (3) classified the reports in the most specific way unless such specificity dramatically reduced the number of reports associated with each individual substance; (4) distinguished the reports associated with plant and fungi and their isolated psychoactive compounds; (5) only included substances with more than 10 reports. This resulted in 165 substances that were manually classified into the following classes: serotonergic psychedelics, dissociative psychedelics, entactogens, deliriants, depressants/sedatives, stimulants, antipsychotics/antidepressants, oneirogens and others. Some substances were also given a secondary category due to the complex nature of their effects.

The data in the Erowid corpus is summarized with detail in the supplementary material. Table S1 presents the following information for serotonergic psychedelics: generic drug names/botanical species, PubChem CID (for molecular compounds) (Hähnke, Kim, \& Bolton, 2018), number of reports and a brief description of the known mechanism of action and the geographic distribution (for naturally occurring plants, mushrooms and cacti) (Rätsch, 2005). Table S2 contains the same information for dissociatives, entactogens and deliriants, Table S3 for depressant/sedatives, Table S4 for stimulants, others and oneirogens, and Table S5 for antipsychotics/antidepressants.

Following previous work, drug categories were determined using a hybrid criterion based on pharmacological action and the subjective effects induced by the substances. Serotonergic psychedelics (5- $\mathrm{HT}_{2 \mathrm{~A}}$ agonists) were grouped based on their mechanism of action (Nichols, 2016). The category of dissociative psychedelics comprised primarily NMDA antagonists such as the arylcyclohexylamines ketamine and PCP, but also included substances with other mechanisms of action (i.e. Amanita muscaria mushrooms) (Wallach \& Brandt, 2018). Deliriants were in most cases Solanaceae plants rich in tropane alkaloids (El-Dabbas \& Evans, 1982). Entactogen drugs were categorized by their subjective effects (Nichols, 1986). Stimulants included dopaminergic drugs such as cocaine, amphetamines and modafinil, as well as others of different pharmacological profile; analogously, depressants/sedatives were defined by their effects on the central nervous system, and included substances such as benzodiazepines as well as natural and synthetic opioid analgesics (Perrine, 1996). Prescription antidepressants and antipsychotics were grouped together into one category. Only two plants in the Erowid corpus are consumed primarily for their oneirogen effect (Calea zacatechichi and Silene undulata) (Toro \& Thomas, 2007). Certain substances had a large number of subjective reports, but their relatively unique mechanism of action did not justify the creation of a new category; such substances were classified as "other" (e.g. Salvia divinorum).

\subsection{Corpora preprocessing}

The preprocessing of the NDE and Erowid corpora was performed using the Natural Language Toolkit (NLTK, http://www.nltk. org/) in Python 3.4.6. We applied the following steps: (1) separation into individual words after discarding all punctuation marks (word repetitions allowed); (2) word lemmatization using NLTK (i.e. conversion to the root from which the word is inflicted); (3) conversion to lowercase; (4) discarding words containing less than three characters (after lemmatization) (Sanz et al., 2018). Since texts from the Erowid corpus are likely to be influenced by the nature of the substance being reported, we manually compiled a list of words including substance names, different slang variations (e.g. "weed", "coke"), and words relating to the possible routes of administration (e.g. "smoke“, "snort", "inject"). These were removed from both the NDE and Erowid corpora. The rationale behind this "censoring" of the corpora was to retain words describing the experiences elicited by the substances, but not the drugs themselves nor contextual factors such as the route of administration.

\subsection{Latent semantic analysis}

We employed an index of semantic similarity between narratives as a proxy of the similarity of the associated altered states of consciousness (Landauer et al., 2004). The main assumption behind our method is that if two subjects are asked to describe certain experiences, and both narratives mention the same core concepts with similar frequencies, then it is likely that both narratives reflect similar subjective experiences. Previous work has established the adequacy of LSA to classify the reported subjective effects of 
different psychoactive substances (Bedi et al., 2014; Coyle, Presti, \& Baggott, 2012), and to compare drug-induced altered states of consciousness vs. non-ordinary experiences of a different nature (Bedi et al., 2014; Sanz et al., 2018).

As a first approximation, we define a matrix $A$ with $T$ rows (number of terms appearing in the corpus) and $D$ columns (number of documents, in this case each document corresponds to all the reports of a substance in the Erowid corpus, or to all the narratives in the NDE corpus). This term-document matrix contains in its $i, j$ entry the number of times the $i$-th term is mentioned in the $j$-th document. Then, it is possible to compute the similarity between documents $j_{1}$ and $j_{2}$ as the linear correlation between their corresponding columns in the matrix $A$ (i.e. the documents are considered similar if the usage of terms is correlated between documents) ${ }^{2}$. A problem with this approach is only counting term occurrences, instead of computing their frequency weighted by how many times the term occurs within a given document vs. across the whole corpus. Thus, we applied the frequency-inverse document frequency (tf-idf) transform, as implemented in scikit-learn (www.scikit-learn.org). The entries of the matrix $A$ are determined as the product of the term frequency and the inverse document frequency. The term frequency is defined as the number of times the term appears in each document. The inverse document frequency is defined as the logarithmically scaled inverse fraction of the documents containing the term. To eliminate very frequent/rare terms from the corpus, only those appearing in more/less than 5\%/95\% of the documents were retained. Intuitively, the tf-idf transform gives a higher weight to terms that are more specific to a given document, decreasing the weight based on the number of documents in the corpus that contain such term.

Another difficulty with the first approximation described above is that, for a large vocabulary of terms, the occurrence frequencies are sparse, i.e. most entries in the matrix $A$ are zero and the linear correlation values of the columns are likely to be very low. By grouping words that appear in similar contexts as concepts, the number of independent variables used to span the semantic space is reduced. For this purpose, we applied LSA, a NLP tool based on the hypothesis that words with similar meaning appear with similar frequency in texts (Landauer et al., 2004). To apply LSA, the term-document matrix obtained using the tf-idf transform was first decomposed into the product of three matrices using Singular Value Decomposition (SDV) (Huang \& Narendra, 1975), $A=U \times \sum \times W$. In this decomposition $U$ and $V$ are real unitary matrices and $\sum$ is a diagonal matrix of singular values ordered by size. To reduce the dimensionality of the semantic space, only the first $k$ singular values of $\sum$ were retained, yielding the truncated matrix $\sum_{k}$. From this matrix, the rank-reduced term-document matrix is computed as $A^{*}=U_{k} \times \sum_{k} \times W_{k}$. In the supplementary material we provide an example of the LSA algorithm applied to a small corpus.

\subsection{Principal component analysis and automated component labeling}

We applied principal component analysis (PCA) to reduce the term-document matrix into a smaller number of components capturing topics that recurrently appear in the corpora (using an algorithm based on SVD as implemented in MATLAB 2014). We considered only the topics represented by the first five components, in terms of explained variance in the tf-idf rank-reduced termdocument matrix. Each component was associated with one coefficient per term in the vocabulary. These coefficients were used to represent the topics in word cloud format using the website https://wordart.com/.

To avoid the arbitrary naming of the components, for each of them we selected the 20 terms with the largest coefficients, and queried a large space for the words with the highest semantic proximity. For this purpose we used the Datamuse API (https://www. datamuse.com/), a word-finding engine based on word2vec (Minarro-Giménez, Marín-Alonso, \& Samwald, 2014), an embedding method using neural networks to map words into a vector space with the constraint that words appearing in similar contexts are also close in the vector space embedding.

\section{Results}

\subsection{Similarity between NDE and drug-induced experiences}

We performed a SVD of the term-document matrix, grouping as single documents all NDE narratives and all reports associated with the 165 substances in the Erowid corpus. We retained the first 20 singular values; this choice was based on the number of drug categories included in the corpus, as well as on previous research showing adequate results using the same dataset (Sanz et al., 2018). The robustness of the results against the choice of this parameter was evaluated and is presented in a latter section.

We obtained the correlation matrix derived from computing all pairwise correlations between the columns of the rank-reduced term-document matrix $A^{*}$ (Fig. 2A). The narratives of substances within the same category tended to display a higher semantic similarity when compared to those of other categories; this was especially clear for antipsychotics and deliriants. Stimulants and sedatives presented a smaller degree of within-group semantic similarity. Reports associated with the use of serotonergic and dissociative psychedelics and entactogens presented the highest within-group semantic similarity when considered together, but their differentiation was difficult. Fig. 2B presents the average semantic similarity values for each substance category, and also includes the semantic similarity with NDE reports. It is evident upon direct visual inspection that NDE reports show the highest degree of semantic similarity with narratives of dissociative drug experiences.

We then computed the semantic similarity between NDE narratives and those associated with each single substance. Fig. 3

\footnotetext{
${ }^{2}$ It must be noted that the similarity is measured in terms of word co-occurrences, and not in terms of the frequency of single words. For instance, the word "fear" could appear frequently in a report of a frightful experience, but also in a report emphasizing absence of fear. However, the different co-ocurrence frequencies of "fear" with other words will increase the semantic distance of the reports.
} 


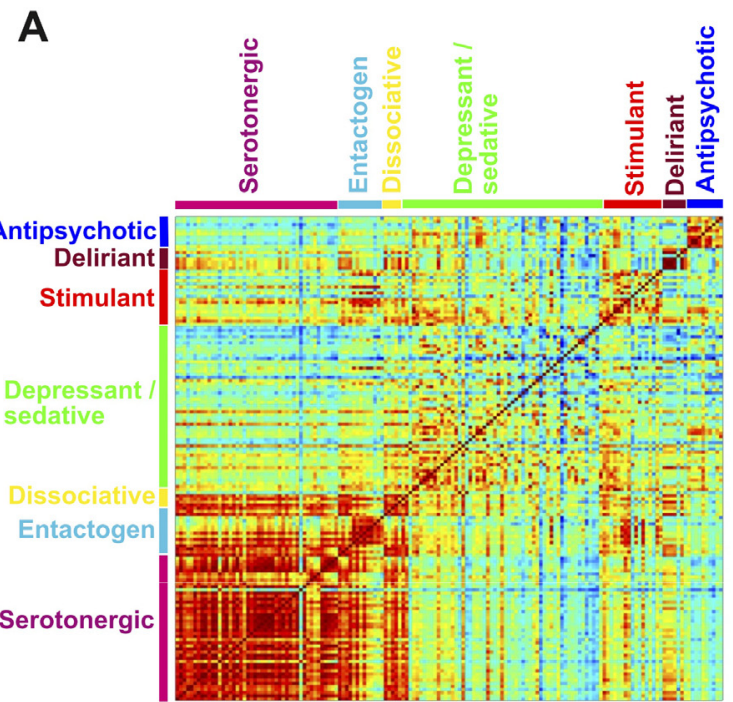

B

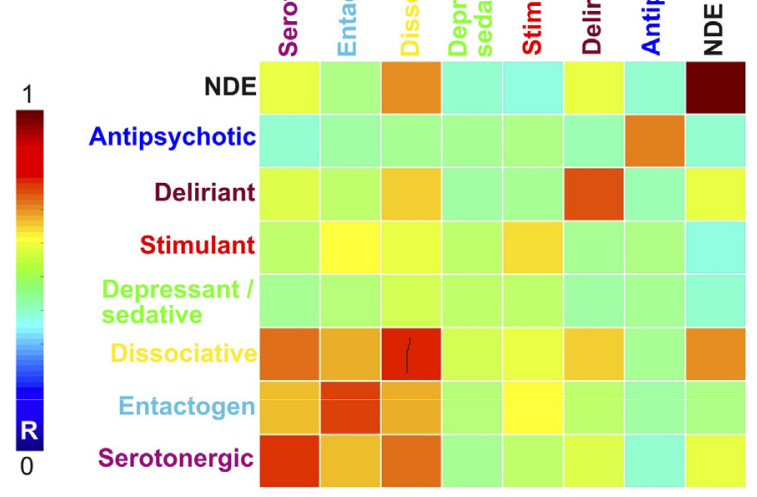

Fig. 2. A. Pairwise document semantic similarity matrix obtained after applying LSA to the term-document matrix and retaining the 20 largest singular values. B. Matrix containing the average similarity values between all documents in each substance class, including the similarity with NDE narratives.

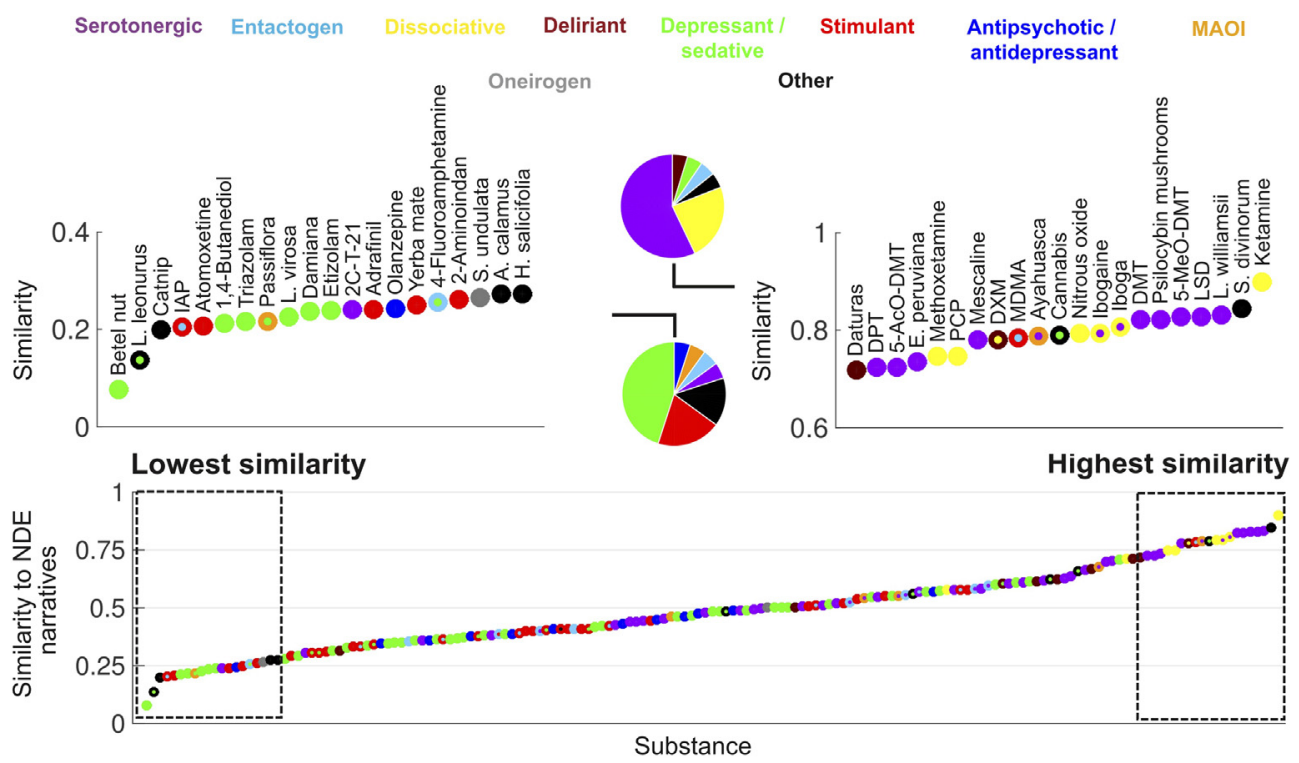

Fig. 3. Ranking of psychoactive substances in the Erowid corpus in terms of the similarity of their reported subjective effects to NDE narratives. The rectangles on the left/right zoom into the top 20 lowest/highest ranking substances. The pie charts indicate the proportion of each primary category within both rectangles. Substances are represented with circles that are color-coded based on their category (the color of the center/border corresponds to the primary/secondary category).

presents a ranking of the substances in terms of such similarity. Each circle represents a drug that is color-coded based on its primary (center) and secondary (border) category. The rectangles in the left/right of the figure zoom into the 20 substances whose reports present the lowest/highest semantic similarity to NDE narratives. The pie charts indicate the proportion of each drug category in the top/bottom ranked substances. While the most represented category among the top 20 substances was that of serotonergic psychedelics, the dissociative psychedelic ketamine presented the highest similarity to NDE reports, followed by $S$. divinorum, and a series of naturally occurring (L. williamsii, 5-MeO-DMT, Psilocybe spp., DMT, iboga, ibogaine, ayahuasca, mescaline and E. peruviana), semi-synthetic (LSD) and synthetic (5-AcO-DMT, DPT) serotonergic psychedelics. Other dissociatives that ranked among the top 20 substances were nitrous oxide, PCP and methoxetamine. The only deliriants were the plants of the Datura genus. Conversely, the 20 substances with the lowest similarity to NDE narratives only included one serotonergic psychedelic (2C-T-21), and neither deliriants nor dissociative psychedelic. Most of the substances were either sedatives or stimulants.

The boxplots in Fig. 4A show the average ranking (in terms of semantic similarity to NDE narratives) for each substance category. 
A

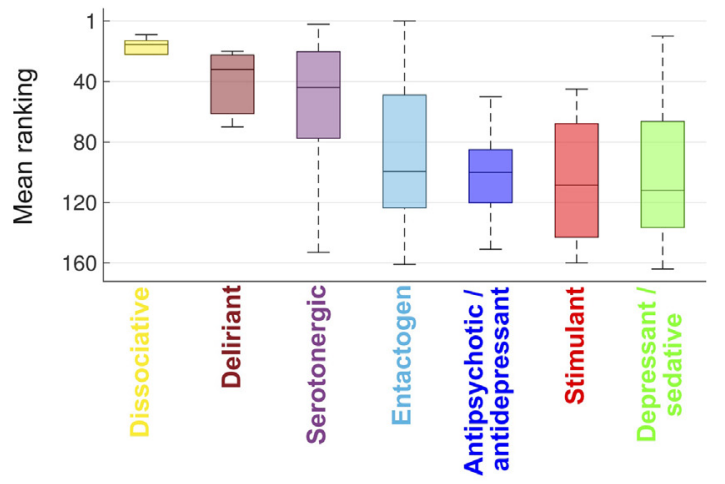

B

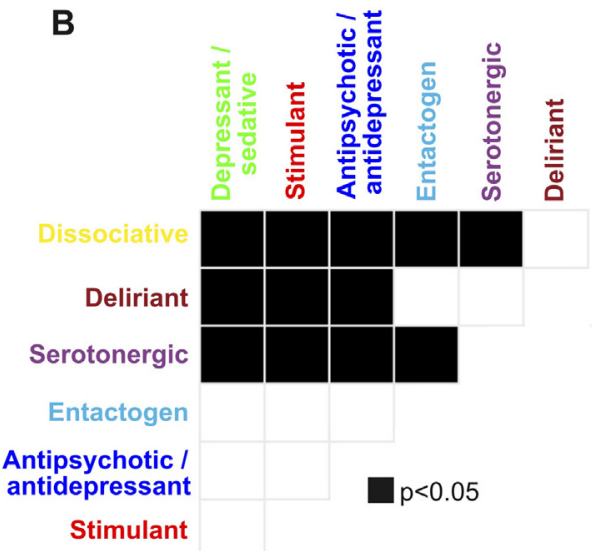

Fig. 4. A. Average substance ranking per category (in terms of the similarity of the reported effects to NDE narratives). Boxplots indicate median \pm 25 th and 75th percentiles. Lower numbers represent higher semantic similarity (i.e. the highest possible rank is 1 , corresponding to ketamine). B. Pairwise Wilcoxon signed-rank tests for the difference in the semantic similarity to NDE narratives for each pair of drug categories. Black squares indicate a significant difference $(\mathrm{p}<0.05)$ for the pair of drug categories in the corresponding row and column.

Dissociatives ranked first, followed by deliriants and serotonergic psychedelics. Pairwise Wilcoxon signed-rank tests showed that the mean ranking of dissociative substances was higher than that of all other categories except deliriants. All hallucinogens (e.g. dissociatives, deliriants and serotonergic psychedelics) presented a significantly higher average ranking than sedatives, stimulants and antipsychotics/antidepressants.

\subsection{Lexical and semantic similarities between NDE and drug-induced experiences}

We then investigated the lexical and semantic features that related to the similarity between NDEs and reports of drug-induced experiences. We first ranked the terms based on their frequency of occurrence (obtained after applying the tf-idf transform) both in $\mathrm{NDE}$ and ketamine reports. We also computed the average ranking between both sets of reports, to identify those terms having a high prevalence in ketamine and NDE reports. Results are shown in Fig. 5. The most common terms in both sets of narratives relate to perception ("face", "vision", "saw"), emotion ("fear"), consciousness and cognition ("reality", "moment", "universe", "understand", "consciousness", "memory", "explain“, "learn"), the self and others ("own“, "arm“, "self", "person", "human", "everyone“, "others") and setting ("door", "floor", "inside", "outside").

The comparison of the most frequent terms in both sets of experiences suggests shared phenomenological features associated with an altered state of perception of the self and the environment, and a departure from the everyday contents of conscious mentation. The application of PCA allowed us to classify these terms into recurrent topics appearing throughout both corpora. Fig. 6A shows word clouds representing the first five principal components, which cumulatively described more than $60 \%$ of the variance in the data. The terms were weighted by the absolute value of their coefficients in the linear combination required to span each component. The names of the components were obtained by selecting words with the lowest semantic distance to the 20 most relevant terms in each component (using a trained word2vec model available in the Datamuse API, https://www.datamuse.com/). The first five principal components were the following:

1st: "Look/self", included many of the shared terms between NDE and ketamine experiences (Fig. 5), relating to perception, consciousness, and setting.

2nd: "Tone/negative", placed a greater emphasis on negative bodily sensations (i.e. "body load").

3rd: "Make/stuff", included terms related to the preparation of natural materials with the purpose of extracting and isolating their psychoactive compounds.

4rd: "Take/dependency", included terms clearly related to drug abuse, addiction and associated circumstances.

5th: "Disease/religion", included terms related to diseases and their medical treatment, as well as others related to ceremonies, rituals and "spiritual" healing.

In Fig. 6B we show the projection of each category of drugs into the five principal components, as well as the projection of the NDE narratives. The radar plots present "fingerprints" consistent with the known subjective and physiological effects of the drugs. For instance, serotonergic psychedelics projected almost exclusively into the "look/self" component, stimulants into the "take/"tone"/ negative" components (likely a consequence of their medical use and associated dependency" and "tone/negative" components, and antipsychotics into the "disease/religion" and physical side effects). Deliriants induce profound alterations in consciousness and are generally prepared from plant materials (e.g. Datura spp., Brugmansia spp.) (El-Dabbas \& Evans, 1982), thus having the largest projections into the "look/self" and "make/stuff" components. NDE narratives projected into three different components: "look/self", "disease/religion" and "make/stuff". 

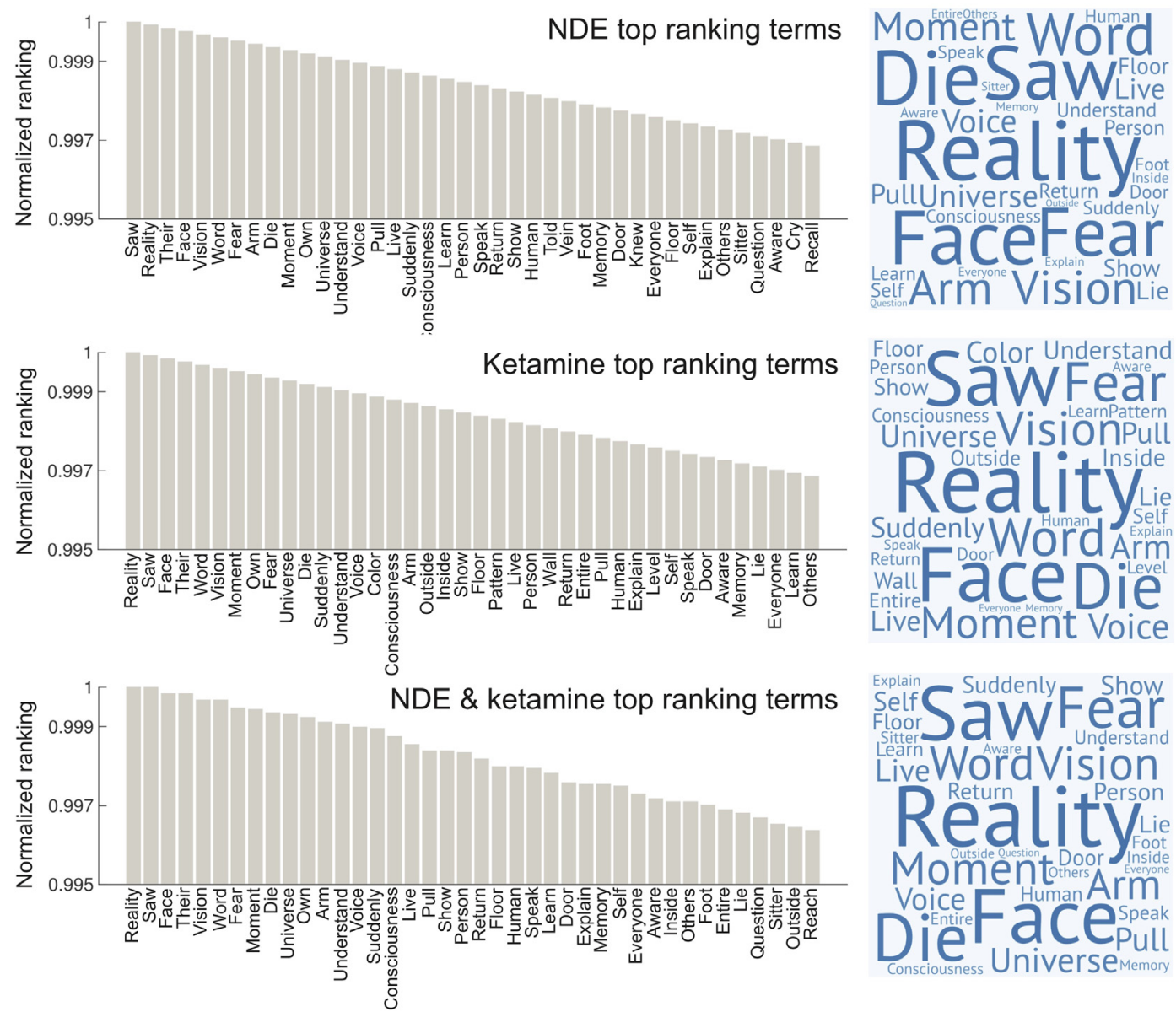

Fig. 5. Lexical similarity between NDE and ketamine reports. The upper panel presents the 40 most frequent terms in the NDE narratives, together with a word cloud representation (word size was weighted by the normalized ranking). The middle panel presents the same information for narratives of ketamine-induced experiences, while in the bottom panel terms are ordered based on the average frequency across both sets of narratives.

To visualize the relationship between the semantic similarity of NDEs and the narratives associated with specific psychoactive substances, we superimposed the radar plots in Fig. 6C. This comparison was done for the projections of NDEs vs. ketamine, DMT, cocaine and heroin reports. For the first two substances, the similarity with the NDE semantic "fingerprint" is apparent upon direct inspection, while for the other two substances obvious differences are manifest, mainly involving their projections into the "take/ dependency“ component.

\subsection{Robustness vs. number of singular values retained}

The SVDs performed in the previous sections retained the 20 largest singular values. In Fig. 7A we show the correlation coefficient matrix for pairs of vectors that contain the rankings of the 165 psychoactive substances obtained using a number of singular values ranging from 5 to 145 . We observe that modest to high correlations appeared near the diagonal of the matrix, indicating that small changes in the number of retained singular values did not dramatically alter the ranking of the substances. The correlations became more stable as the number of singular values was increased. We also note that two ranking vectors computed using a very different number of singular values (e.g. 20 vs. 100) compare poorly. In Fig. 7B we investigate the robustness of substance category ranking vs. number of retained singular values. We observe that consistent results emerged for the highest ranked substances. In other words, while changing the number of singular values influenced the ranking of substances whose reports did not present a high semantic similarity to NDE narratives, those substances with a high semantic similarity to NDE narratives remained relatively stable. When computing the mean similarity across all choices of the number of retained singular values (Fig. 7C), ketamine emerged again as the substance whose reports presented the highest semantic similarity to those of NDE, followed by a series of substances whose ordering is similar to that of Fig. 3. From retaining 5-145 singular values, ketamine ranked first in most instances, followed by LSD, ibogaine, L. williamsii and PCP (Fig. 7D). 
A "Look / self" "Tone / negative" "Make/ stuff"

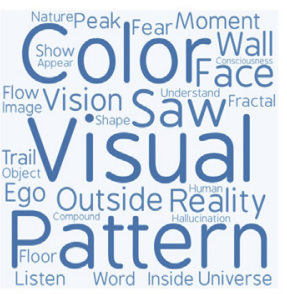

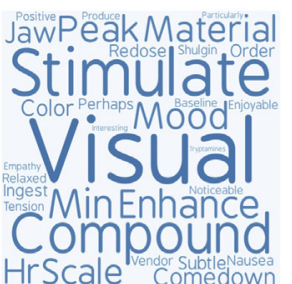

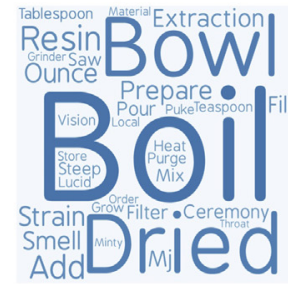

"Take /

vomitTolerance Clean

Withdrawal

Sick ${ }^{\text {raw }}$ Nausea

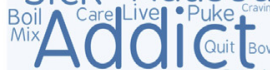

Dealer Money

vein $W$ armirmsell
"Disease /

Anxiet tove Purgear Clinic Ceremony

EgoPrescribe self

Withdrawal

MlMedcation consciousness

Pattern Sorititual. Treatment

Depression sing

voice symptom Universe

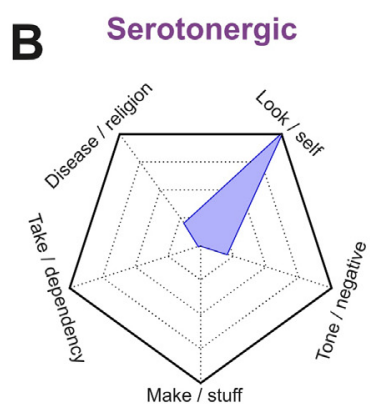

Stimulant

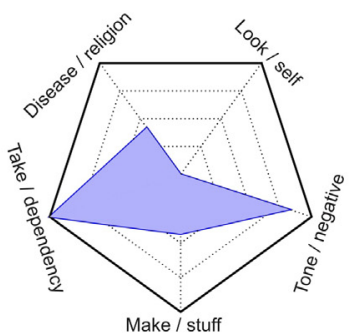

C NDE Ketamine

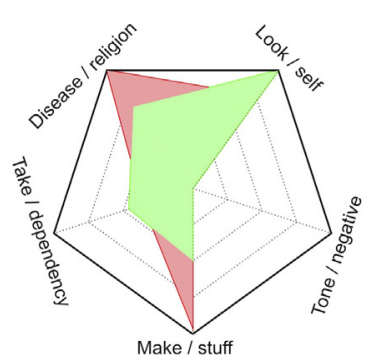

Entactogen

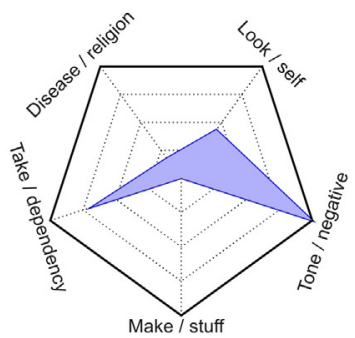

Antipsychotic I antidepressant

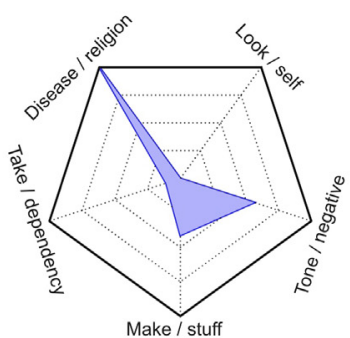

NDE DIMT

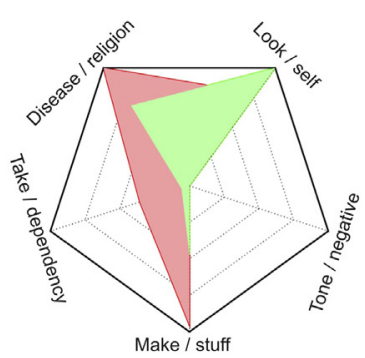

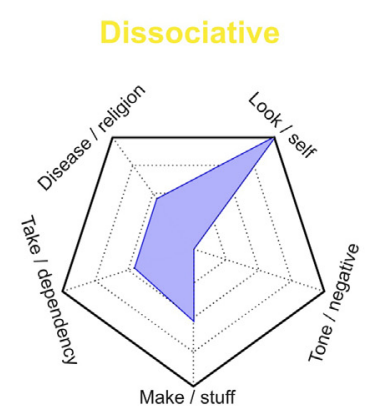

Deliriant

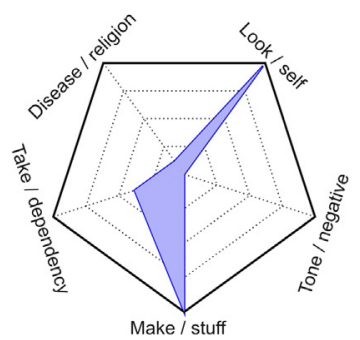

NDE Heroine

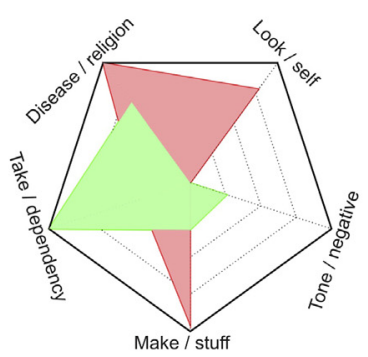

Depressant / sedative

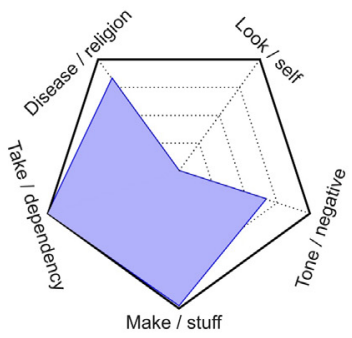

NDE

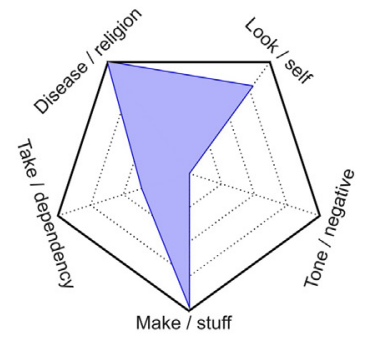

NDE Cocaine

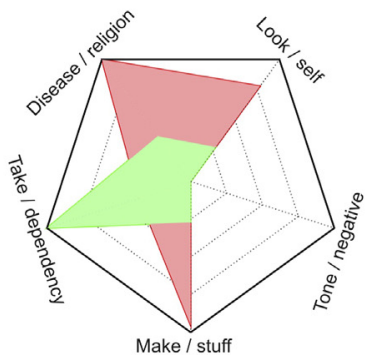

Fig. 6. A. Five first principal components, ordered by decreasing explained variance. B. Radar plots showing the projections of the narratives (per substance category and NDEs) into each of the five principal components. C. Radar plots showing the comparison between the projections of NDE narratives into the five principal components and those of ketamine, DMT, heroin and cocaine narratives.

\subsection{Relationship between semantic similarity and NDE metadata}

We understand as "NDE metadata" all the information that is not contained in the NDE narratives themselves, but informs on the surrounding circumstances and demographics. In Fig. 8A, we show the mean rating (in terms of NDE narrative semantic similarity) for each drug category, when comparing to NDEs associated with loss of consciousness due to head injury, anesthesia/drug use, and cardiac arrest. The mean ranking vs. drug category remained stable across conditions, and resembled that of Fig. 4A. A similar result is shown in Fig. 8B (proximity to death) and Fig. 8C (emotional valence of the experience). As shown in the pie charts, ketamine emerged in almost all cases as the drug whose reports presented the highest semantic similarity to NDE narratives. The only exception 

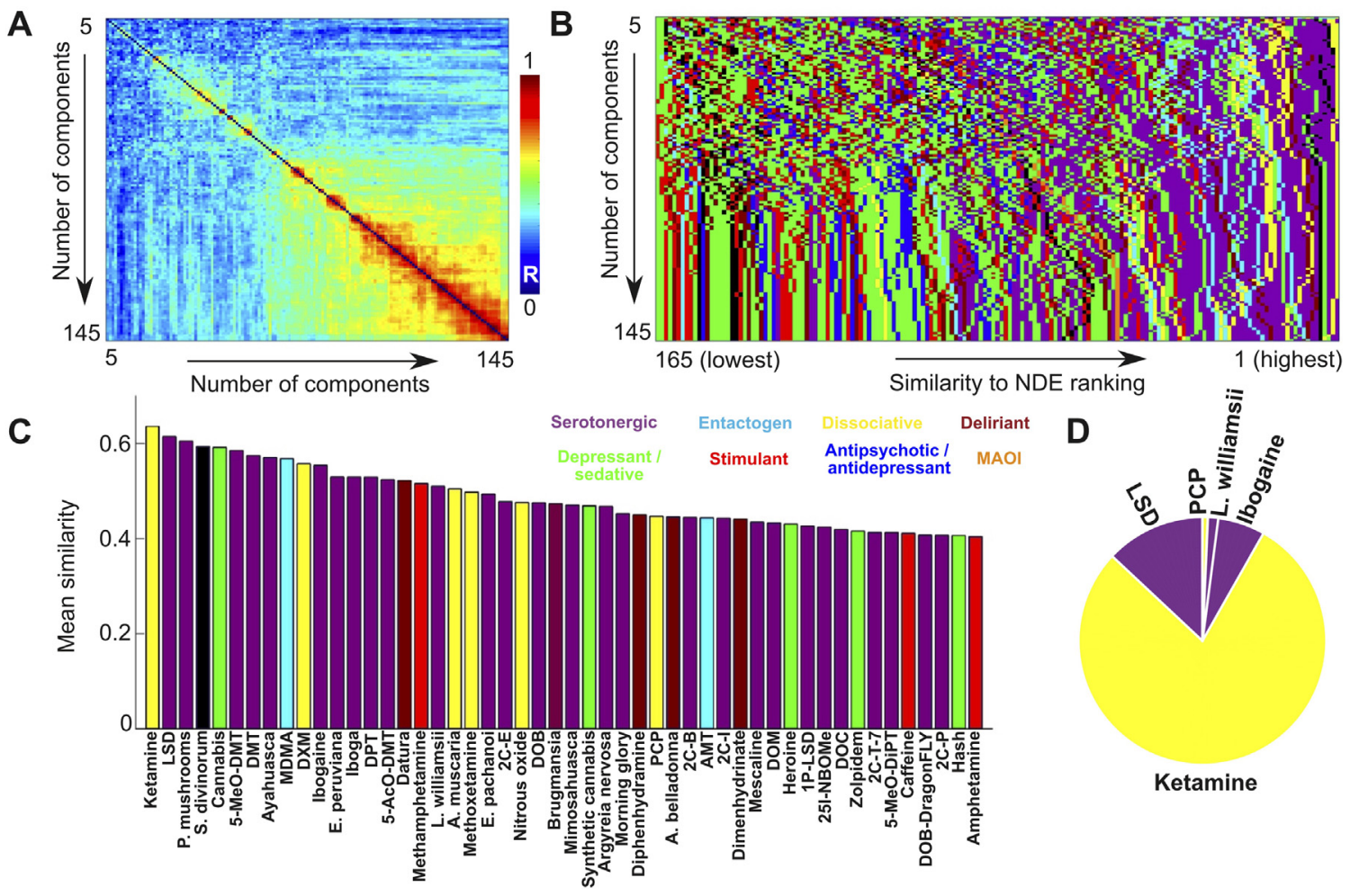

Fig. 7. Robustness vs. the number of singular values. A. Correlation matrix between the vectors that contain the rankings of the 165 substances (in terms of the similarity of their associated reports to those of NDE narratives), when computed using different numbers of retained singular values (from 5 to 145 singular values). B. Semantic similarity to NDE narratives as a function of the number of retained singular values, color-coded by the category of the drug in each ranking position. Substances with lower rankings were not stable against changes of this parameter, while the top ranked substances remained relatively stable. C. Substances ranked by their mean semantic similarity to NDE narratives (computed across all choices of the number of retained singular values, from 5 to 145). D. Pie chart showing the proportion of instances in which a substance ranked first (among all choices of the number of retained singular values).

was the comparison with NDEs associated with loss of consciousness induced by cardiac arrest, for which LSD ranked comparably to ketamine. Statistical analyses (one-way ANOVA) did not detect a significant effect of the NDE metadata on the drug ranking.

\subsection{Replication of results with additional censored terms}

To evaluate whether our main results depended on the presence of the contextual information in the "make/stuff" and "take/ dependency" components, we repeated the analysis after censoring the top 500 most relevant terms of each component. After applying LSA (retaining the highest 20 singular values), the top ten drugs in terms of the semantic similarity of their written reports to those of NDEs were the following: ketamine, ayahuasca, S. divinorum, L. williamsii, 5-MeO-DMT, LSD, Cannabis, Psilocybin mushrooms, iboga, nitrous oxide. Conversely, the ten least similar were: betel nut, atomoxetine, IAP, triazolam, yohimbe, passion flower, 2C-T-21, etizolam, olanzapine, 2C-T-4.

\section{Discussion}

Even disregarding processes at the sub-cellular scale and characterizing the $\approx 10^{11}$ neurons as "on/off" binary units, the number of potential physical states of the brain is astonishing. Such complexity is required to support the highly differentiated nature of consciousness (Tognoli \& Kelso, 2014; Tononi \& Edelman, 1998; Tononi, 2012). Given the diversity in the potential contents of human experience, it is remarkable that those contents can be organized into qualitatively different global states of consciousness. Even more remarkable is that analogies can be made between a finite set of "altered states", whose underlying neurophysiological correlates are seemingly unrelated. Especially interesting are the comparisons between pharmacological and physiological or spontaneously occurring altered states of consciousness, since such comparisons could inform the development of neurochemical models for the latter (Luke, 2008; Strassman, 2001).

We addressed the long-standing analogy between the experience of dying and the acute effects of certain psychoactive drugs. Links between dying, death, a potential existence of afterlife and certain hallucinogenic plants and fungi emerged independently across different societies, and are also ubiquitous in contemporary psychedelic culture. However, scarce empirical research has been 
A

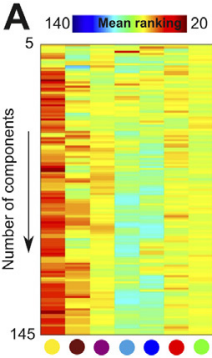

B
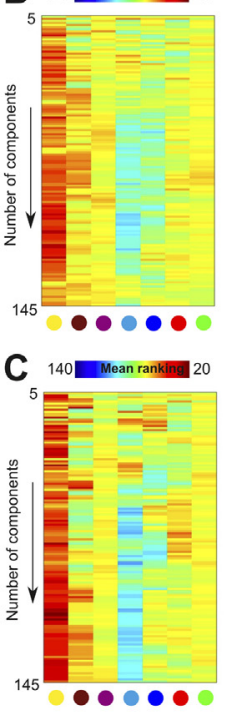

Head injury

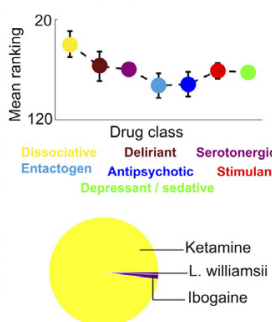

Not serious

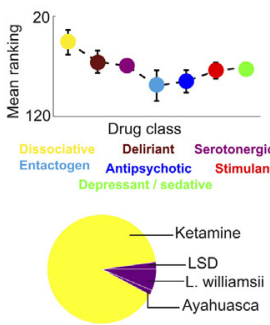

Pleasant

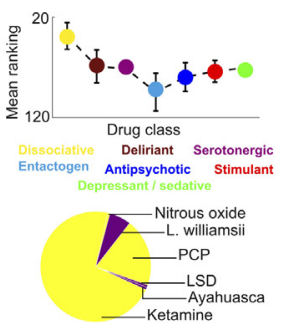

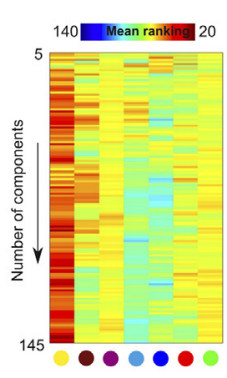

Drug / anesthesia

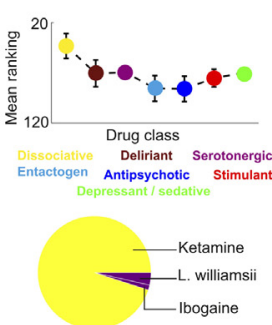

Serious
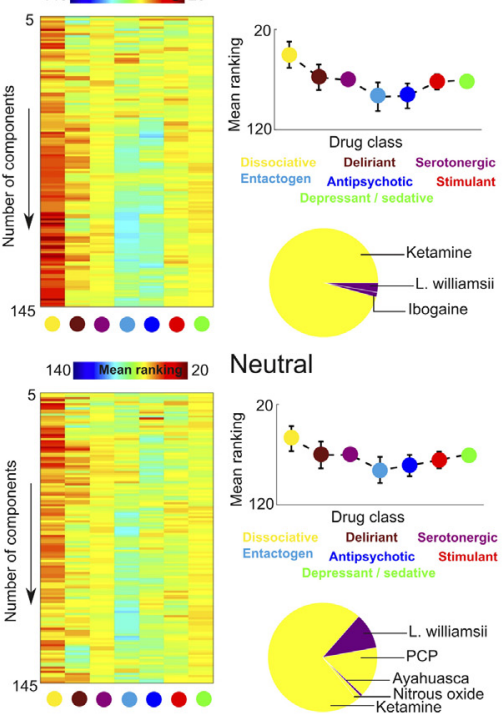

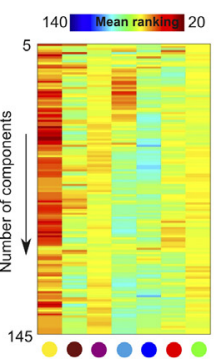

Cardiac arrest

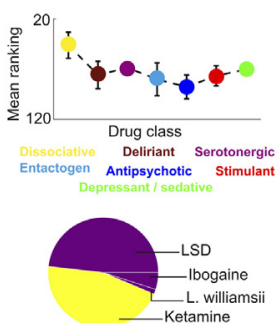

Critical
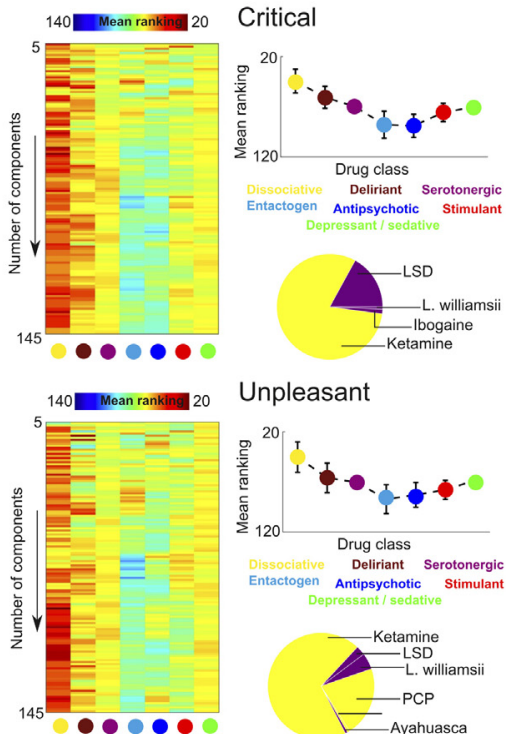

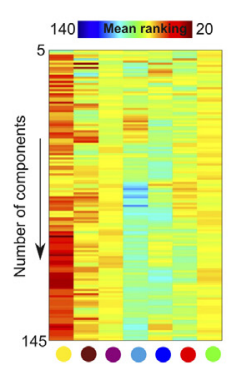

Unpleasant

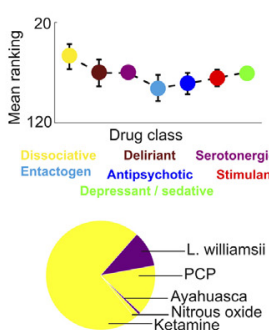

Fig. 8. Similarity between narratives associated with substance use and NDEs of different cause, proximity to death and emotional valence. A. Semantic similarity between drug category and NDEs associated to loss of consciousness due to head injury, anesthetic agents/other drugs, and cardiac arrest. The rows of the matrices indicate the number of singular values retained in the SVD decomposition for LSA, and the columns indicate the drug category (color-coded as in Figs. 3 and 4). The sub-panels include the average ranking per substance category, as well as pie charts showing the proportion of times that the reports of a given substance presented the highest similarity to those of NDE narratives. B. Same information as in panel A, but for NDEs separated by the proximity to death (not serious, serious and loss of vital signs). C. Same information as in panels A and B, but for NDEs separated by the emotional valence attributed by the experiencers (pleasant, neutral, unpleasant).

conducted to clarify the nature of this relationship. Unlike other human experiences, dying is difficult to study under controlled laboratory conditions by means of repeated measurements, which is a necessary condition to demonstrate within- and betweenindividual robustness, and to determine a core set of phenomenological features. Lacking a precise definition, the association between pharmacologically altered states of consciousness and "dying" could represent the cultural propagation of an originally arbitrary association (i.e., "meme") (Dawkins, 2006).

The systematic study of reports of individuals who survived close brushes with death suggests an alternative hypothesis. NDEs present a common core of robust and reproducible phenomenological features with relatively minor variations in terms of cultural background (Belanti, Perera, \& Jagadheesan, 2008), even though certain features (e.g. life review and tunnel sensations) were shown to depend on religious beliefs (Kellehear, 1993), and the robustness of NDEs does not necessarily imply a consistent temporal ordering of the defining phenomenological features (Martial et al., 2017) - although previous works have revealed certain consistencies across cultural backgrounds (Athappilly, Greyson, \& Stevenson, 2006) and in terms of temporal ordering (Lange, Greyson, \& Houran, 2004).

This body of empirical evidence supports that near-death is by itself an altered state of consciousness that can be investigated using quantitative psychometric scales (Greyson, 1983). Two studies followed this approach and compared experiences induced by ketamine (Corazza \& Schifano, 2010) and DMT (Timmermann et al., 2018) to NDE phenomenology, the latter having the merit of assessing DMT phenomenology under controlled experimental conditions including a placebo. These studies support a significant overlap between both altered states of consciousness, yet a comparison including a wider range of substances is needed to systematically evaluate how such overlap depends on the pharmacological mechanism of action of different drugs, as well as on the neurophysiological changes elicited by them. The use of free narratives of drug-induced experiences presents many limitations (see below for a discussion), but nevertheless allows a comparison that is massive both in terms of the investigated drugs and the number of associated reports. 
The comparison between NDEs and drug-induced experiences associated with an ample range of mechanisms of action offers the possibility of indirectly evaluating neurochemical models of NDEs. Strassman developed a parsimonious model for NDEs suggesting a relationship with DMT, a serotonergic psychedelic whose presence is demonstrated in human cerebrospinal fluid (Barker, McIlhenny, \& Strassman, 2012; Strassman, 2001). The author further hypothesized that DMT production primarily occurs in the pineal gland, given its unique location outside the blood-brain barrier and high concentrations of both serotonin and methyltransferase enzymes (Barker, Borjigin, Lomnicka, \& Strassman, 2013), which are required for in vivo conversion of tryptamine to DMT. However, postmortem attempts to isolate DMT from pineal glands were unsuccessful (Strassman, 2001). Regardless of these considerations, both our analysis and the recent work by Timmermann and colleagues (Timmermann et al., 2018) established that DMT-induced experiences present a substantial overlap with NDEs, with especially strong associations between DMT-induced near-death type experiences and mystical-type experiences, and the feelings of "ego-dissolution", "unitive experience" and "oceanic feeling". This study also established associations between NDE-like DMT experiences and certain personality traits (this association with personality traits is also known in other types of NDE-like); in agreement with the observation that both NDEs and psychedelic experiences are highly dependent on contextual factors (Greyson, 1993; Nichols, 2016; Studerus, Gamma, Kometer, \& Vollenweider, 2012). Our study established the similarity of both experiences by means of free narratives. The application of dimensionality reduction techniques (PCA) revealed discrete components with similar ratings between DMT experiences and NDEs, especially in components related to alterations in conscious perception of the environment and the self ("look/self") and therapeutic, mystical and spiritual aspects of the experience ("disease/religion"). These results are consistent with the use of psychometric scales by Timmermann et al. (2018). We must note, however, that it has not been established that the numerical ratings extracted from free narratives are equivalent to those obtained using psychometric scales- even though preliminary analyses have been conducted for the NDE Scale (Lange et al., 2015). Importantly, our analysis revealed that such commonalities were not specific to DMT experiences, but common to other serotonergic psychedelics as well. Among the top 20 substances eliciting experiences most similar to NDEs, more than 50\% of them were serotonergic psychedelics, including L. williamsii, LSD, 5-MeO-DMT and mushrooms of the Psilocybe genus, all of them ranking higher than DMT.

A possible biological role for the release of endogenous DMT at times of stress is related to its neuroprotective effects, in particular, the prevention of cell death due to hypoxia and oxidative stress mediated via $\sigma_{1}$ receptor agonism (Frecska, Szabo, Winkelman, Luna, \& McKenna, 2013; Szabo et al., 2016). These effects remain to be demonstrated in humans; furthermore, Nichols has argued that endogenous DMT production could not reach concentrations sufficiently high to elicit any noticeable changes in subjective experience (Nichols, 2018). Our analysis revealed that ketamine, a synthetic arylcyclohexalamine dissociative anesthetic, was associated with narratives most similar to those of NDEs, regardless of the circumstances surrounding the NDEs, the actual proximity to death and the emotional valence assigned by the experiencers. Ketamine acts by antagonism at the PCP site of glutamate NMDA receptors. Such antagonism has been shown to prevent cell death due to the excitotoxicity caused by hypoxia (Church, Zeman, \& Lodge, 1988; Rothman \& Olney, 1986). Many studies and clinical trials have established that ketamine shows neuroprotective and neuroregenerative effects in humans, even when administered after onset of cerebral insult, improving the clinical outcomes related to stroke, brain injury and status epilepticus (Church et al., 1988; Fujikawa, 1995; Hudetz \& Pagel, 2010; Marcoux, Goodrich, \& Dominick, 1988). This led Grinspoon and Bakalar (Grinspoon \& Bakalar, 1979) and subsequently Jansen (Jansen, 1989, 1997a,1997b) to suggest that an endogenous ketamine-like compound is released at times of stress and is responsible for the remarkable similarities between ketamine-induced experiences and NDEs. Candidate endogenous agents ("endopsychosins") (Jansen, 1990) have been found in animal tissues, but are likely complex neuropeptides and their molecular structure remains to be determined (DiMaggio, Contreras, Quirion, \& O'Donohue, 1986). Other substances suggested as endogenous neuroprotective NMDA antagonists are N-acetylaspartyl-glutamate, kynurenic acid and agmatine (Luke, 2008). The role of glutamate in NDEs is further supported by shared similarity with certain types of epilepsy associated with massive glutamate release (Laureys, Gosseries, \& Tononi, 2016; Morse et al., 1989).

The hypothesis of DMT and endogenous NMDA antagonists as the sole causes of NDEs has received extensive criticism, both in terms of the associated phenomenology and neurobiological plausibility. NDEs can occur in situations in which the threat is only apparent or even absent (e.g. NDE-like phenomenology during hypnagogic or meditative states) and brain cells are not under direct stress (Fenwick, 1997); however, Jansen did not rule out the possibility of an anticipatory NDMA receptor blockade, stating that "there is no reason to suspect that the NDE mechanism would never be activated spontaneously" (Jansen, 1997a,1997b) - indeed, our analyses ranked ketamine-experiences as the most similar to NDEs regardless of the proximity of death (Fig. 8B). Furthermore, OBEs are one of the most salient features of ketamine experiences (Wilkins, Girard, \& Cheyne, 2011), which Grinspoon and Bakalar described as “... becoming a disembodied mind or soul, dying and going to another world. Childhood events may also be re-lived. The loss of contact with ordinary reality and the sense of participation in another reality are more pronounced and less easily resisted than is usually the case with LSD. The dissociative experiences often seem so genuine that users are not sure that they have not actually left their bodies" (Grinspoon \& Bakalar, 1979). It has been shown that OBEs are frequent in NDEs associated with cardiac arrest (French, 2005), as well as in other kinds of NDEs (Charland-Verville et al., 2014), supporting a link to NMDA receptor blockade via an endogenous ketaminelike compound.

Differences in NDE and ketamine-induced phenomenology have been noted, especially between the generally blissful nature of NDEs and the potential occurrence of ketamine "bad trips" (Fenwick, 1997; Strassman, 1997). This objection can be countered by noting that the blissful nature of NDEs may have been overestimated (Greyson \& Bush, 1992), that contextual factors ("set and setting") play an important role in the emotional valence of NDEs and ketamine experiences (Greyson, 1993; Grinspoon \& Bakalar, 1979) and finally noting that the subjective effects elicited by an endogenous NMDA antagonist do not need to exactly match those of ketamine (unless that antagonist is ketamine itself). In support of the ketamine model, it has been argued that subjects who 
experienced both NDEs and ketamine report a remarkable similarity between both experiences (Jansen, 1997a,1997b); however, lacking proper quantification, it is difficult not to dismiss this evidence as anecdotal (Strassman, 1997). Further criticism to the ketamine model is based on the observation that not all individuals in the proximity of death actually report an NDE (Sleutjes et al., 2014); however, a similar percentage of the population is unable to recall their ketamine experiences (Jansen, 1997a,1997b). Conversely, neurochemical models based on other serotonergic psychedelics received criticism due to the divergence between typical psychedelic imagery and the hallucinatory content present during NDEs. Nevertheless, DMT is known to produce more immersive experiences with intense imagery that can include tunnel-like visions and encounters with seemingly autonomous "entities" (Strassman, Qualls, Uhlenhuth, \& Kellner, 1994; Strassman, 2001). The strong association between ketamine and OBEs is shared with NDEs, but is less manifest in the case of serotonergic psychedelics, including DMT (Wilkins et al., 2011).

Our analysis included reports from a large number of substances and thus allowed the exploration of drugs whose phenomenology is not commonly associated with that of NDEs. While on average the phenomenology of substances classified as dissociatives was the most similar to NDEs (with significantly higher similarity than that of serotonergic psychedelics), reports associated with the use of deliriants also presented a high semantic similarity to NDE narratives. These comprise plants of the Datura and Brugmansia genera, which are rich in alkaloids of anticholinergic effect known to induce complex and interactive hallucinations, a general state of confusion and anterograde amnesia (El-Dabbas \& Evans, 1982; Iglesias-Lepine, Mariñosa, Ferrer-Da Pena, \& Epelde, 2012). Drug users frequently lack the insight to identify the imagery as hallucinatory. Analogously, individuals who report NDE are also known to believe in the reality (i.e. corresponding to appropriate real external stimuli) of the experienced events (Schwaninger, Eisenberg, Schechtman, \& Weiss, 2002) due to the experienced clear sensorium. However, since the tropane alkaloids present in these plants are toxic, it is possible that the semantic similarity was driven by descriptions of real life-threatening situations.

Similar considerations may apply to Salvia divinorum, a very potent hallucinogen that is considered safe, but is known to be occasionally smoked by users who believe are consuming cannabis (Vohra, Seefeld, Cantrell, \& Clark, 2011). This plant contains salvinorin A, a highly selective and potent agonist of the $\kappa$ opioid receptor (Chavkin et al., 2004; Roth et al., 2002) capable of producing intense visual, auditory, somatic and vestibular distortions (Addy, Garcia-Romeu, Metzger, \& Wade, 2015; Sumnall, Measham, Brandt, \& Cole, 2011). The intensity of the experience elicited by this drug relative to cannabis may represent a shock to unsuspecting users, who could retrospectively report the belief of being close to death. Nevertheless, it is interesting to note that opiate antagonists (which block the subjective effects of salvinorin A) (Sheffler \& Roth, 2003) such as naloxone can increase survival time in fatal or near-fatal circumstances (Holaday \& Faden, 1978), leading to the hypothesis that certain endorphins (mainly betaendorphin, known to increase in concentration of bodily fluids of dogs moments before their death) (Sotelo, Perez, Guevara, \& Fernandez, 1995) can mediate NDE-like phenomenology (Carr \& Prendergast, 1981). However, beta endorphins present high selectivity for $\mu$ opioid receptors, whose activation, unlike $\kappa$ receptors, is implicated in processes such as analgesia, sedation and euphoria, but not in the production of hallucinations (Loh, Tseng, Wei, \& Li, 1976). Certain endorphins (e.g. dynorphin) bind preferentially to $\kappa$ receptors (Chavkin, James, \& Goldstein, 1982), but there are no experimental results that link them directly to NDEs. The endorphin model of NDEs also presents limitations stemming from the fact that agonism at opioid receptors can produce effects that do not resemble the phenomenological features of NDEs (Sivanesan, Gitlin, \& Candiotti, 2017).

It is interesting to note that the PCA decomposition of the narratives yielded similar "fingerprints" for DMT, ketamine and NDEs (Fig. 6), in contrast to other two control substances (cocaine and heroin). This was mainly due to the fact that these two hallucinogens presented a relative high projection into the "look/self" and "disease/religion" components. The first likely reflects the departure from ordinary conscious perception characteristic of these drugs and NDEs (also manifest in the lexical analysis presented in Fig. 5). With respect to the "disease/religion" component, both ketamine and DMT (as part of the Amazonian concoction ayahuasca) have been explored for therapeutic uses, especially for the treatment of major depression (Kyzar et al., 2017; Palhano-Fontes et al., 2018; Sattar et al., 2018). The presence of words related to spiritual and religious contexts suggests that the potential therapeutic benefits of these substances appear intertwined with ceremonial use in the Erowid corpus (Dakwar et al., 2014; Niciu et al., 2018). This suggestion is supported by the evidence that dissociative NDEs at the time of trauma may provide some therapeutic benefit in reducing the risk of full-blown post-traumatic stress disorder (Greyson, 2001). NDEs are known to produce long-lasting effects, some of which are deemed positive by the experiencers, such as changes in their personal understanding of life, self, and personal relations, modifications in their social customs as well as in their religious or spiritual beliefs and their interest in material possessions and social status (Bianco, Sambin, \& Palmieri, 2017; Dougherty, 1990; Greyson, 2006; Kellehear, 1990; Parnia, Spearpoint, \& Fenwick, 2007; Royse \& Badger, 2017). Increased psychological well-being and self-worth have been linked to NDEs (Noyes, 1980). It is interesting to note that psychedelics were shown to induce similar long-term effects (Forstmann \& Sagioglou, 2017; Gasser, Kirchner, \& Passie, 2015; Griffiths et al., 2016; Grob et al., 2011; Lyons \& Carhart-Harris, 2018). These commonalities lend support to Kenneth Ring's early speculation on the "universality" of transcendental experiences: "The authenticity of a transcendental experience is revealed by its transformative effects (...). My reading of the evidence suggests that whether the trigger be a spontaneous mystical experience, a psychedelic episode, or an NDE, once this core is activated, it begins to unfold and bring about transformation in much the same way, as if an archetype of transformation were engaged" (Ring, 1988). However, it must also be noted that both psychedelics and NDEs can be distressing experiences with negative sequelae. The changes in personal beliefs can be sudden and disorienting, and feelings of anger, depression and estrangement from family and friends have been reported (Duffy \& Olson, 2007; Greyson, 1997). There have also been symptoms of post-traumatic stress disorder (PTSD) associated with NDEs, even though avoidance symptoms are less prevalent, leading to the suggestion that the positive affect linked to NDE-dissociative episodes mitigate PTSD symptoms that frequently arise from other dissociative experiences (Greyson, 2001). Overall, it is clear that both NDEs and certain hallucinogenic drugs with similar phenomenology, such as ketamine and serotonergic psychedelics, can induce long-lasting effects on personality and well-being, but further research is needed to precisely determine the nature of these effects and their prevalence. Finally, NDE reports also presented 
a significant projection into the "make/stuff" component, likely representing contextual information and the description of activities not directly related to the NDEs. Nevertheless, the similarity with the reports of ketamine-induced experiences remained the highest even after censoring the 500 most relevant terms of the "make/stuff" and "take/dependency" components.

Neurochemical models of NDEs can be theoretically attractive; however, we must emphasize that the analyses conducted in the present work neither validate nor refute these models, since they are based on retrospective reports and no physical examinations of the subjects at the time of the reported NDEs were performed. Regardless of the validity of different neurochemical models of NDEs, a very interesting question was posed by R. Strassman: "if so, so what?" (Strassman, 1997). In other words: even if the validity of one of these models is confirmed, why is neuroprotection linked to substances such as ketamine-like compounds and/or DMT, which are also known to elicit an altered state of consciousness frequently characterized by feelings of bliss and transcendence? Why is neuroprotection by endogenous substances not associated with unconsciousness, or with feelings of distress and aversion? It has been shown that decreased fear of death can be a long-term consequence of NDEs (Greyson, 2007a,2007b); thus, NDEs seemingly lack adaptive value. However, this conclusion relies on the assumption that decreased fear of death leads to less caution or increased risktaking behavior. Also, it could be argued that elderly individuals are more likely to experience health problems leading to NDEs, and that feelings of aversion towards death could result in the prolonged competition for resources with individuals who have more potential to reproduce. Furthermore, NDE-dissociative episodes might mitigate PTSD symptoms linked to other dissociative experiences (Greyson, 2001) and NDE experiencers seem to endorse more anti-suicidal attitudes as compared to non-NDE experiencers who have come close to death (Greyson, 1993), thus adding support to the potential adaptive value of these experiences. In any case, drugs that mimic NDE phenomenology might also reduce death-related anxiety, suggesting a potential therapeutic use for the terminally ill. Evidence exists that certain drugs whose reported subjective effects present a high semantic similarity to NDE reports can alleviate end-of-life anxiety in patients with advanced stage cancer, i.e. psilocybin, LSD and ketamine (Griffiths et al., 2016; Grob et al., 2011; Kolp, 2007).

Given the nature of our analyses, our discussion is biased towards the discussion of neurochemical models of NDEs. However, unless direct empirical evidence is obtained, we can only affirm that the reported phenomenology of certain drugs is similar to that of NDEs, and that those drugs could be effective tools for the safe and temporary induction of NDE-like altered states of consciousness in controlled laboratory settings. It is nevertheless certain that these laboratory-induced NDEs may be a mere "reflection" of "authentic" NDEs. Like all models, those based on the effects of psychoactive drugs present limitations. For instance, certain effects of subanaesthetic doses of ketamine are not among the features that characterize NDEs, arguing against the reductionist explanation of NDEs as a consequence of NMDA receptor blockade (Adler et al., 1999; Dillon, Copeland, \& Jansen, 2003). Other models have been proposed that are not directly related to endogenous psychoactive compounds, but to alterations in neural activity in certain brain areas, primarily in temporal lobe regions (Britton \& Bootzin, 2004; Saavedra-Aguilar \& Gómez-Jeria, 1989). Multifactorial explanations for NDE phenomenology have also been proposed. Mobs and Watt argued that each "classic" feature of this phenomenology can be explained via a different mechanism, e.g. awareness of being dead as a form of Coward syndrome, OBEs as a failure to integrate multisensory information, the perception of a tunnel of light as a consequence of retinal ischemia, etc. (Mobs \& Watt, 2011). However, it has been pointed out by Greyson and colleagues that this article only accounted for those features that could be explained by the authors, while ignoring other features that are regarded as the most defining for NDEs (Greyson, Holden, \& van Lommel, 2012). Furthermore, these alternative models must also be understood as speculative, given the lack of experiments performed at time of reported near death. Few experiments have investigated the neurophysiological changes associated with the ceasing of vital functions. Most strikingly, a surge of coherent high frequency (gamma, $>25 \mathrm{~Hz}$ ) oscillations preceding isoelectric activity has been demonstrated both in humans and animal models (Borjigin et al., 2013; Chawla et al., 2017). However, Greyson and colleagues provided a series of arguments against the interpretation of these short surges of "hyperactivity" as neural correlates of NDEs (Greyson, Kelly, \& Dunseath, 2013). It is nevertheless important to note that serotonergic psychedelics such as LSD and psilocybin result in broadband reductions of brain oscillations (Carhart-Harris et al., 2016; Muthukumaraswamy et al., 2013), while ketamine enhances gamma oscillations in parietal and cingulate cortices and medial frontal theta oscillations, and decreases occipital, parietal and anterior cingulate alpha power (Lazarewicz et al., 2010; Muthukumaraswamy et al., 2015). While these results suggest commonalities (alpha power decreases) and divergences (gamma power increases) between the spectral changes elicited by ketamine and serotonergic psychedelics, muscle artifacts are known to heavily affect high frequency bands under the effects of the latter (Muthukumaraswamy, 2013), and increased gamma oscillations have been reported for ayahuasca and LSD (Muthukumaraswamy \& Liley, 2018; Sattar et al., 2015, Schenberg et al., 2015). It is therefore difficult to compare the spectral changes elicited by serotonergic psychedelics and ketamine, and those recorded during the cease of vital functions.

Our study presents both advantages and limitations stemming from the use of a large number of retrospective narratives. In both cases, these narratives rely on the memory of the event. The time between the occurrence of the NDEs and participation in the study averages approximately two decades (Greyson, 2007a,2007b). Reports of subjective effects from Erowid Experience Vaults are likely closer to the time of substance use, but present several limitations of their own. While substantial relevant data was available for the participants of the NDE study, demographical information is not commonly available for Erowid users. Reports could be contaminated by expectation effects, and most lack laboratory verification of the identity and dose of the consumed substances, which may be even unknown to the users reporting the experiences. Dose could bias the results if subjects consumed higher doses of ketamine compared to those of serotonergic psychedelics such as LSD and psilocybin, resulting in dissociation and feelings of disembodiment only for the latter. However, DMT is known to produce these effects at relatively low doses when smoked or administered intravenously (Strassman et al., 1994; Winstock, Kaar, \& Borschmann, 2014), and ketamine reports still presented a higher semantic similarity to those of NDEs than the DMT reports. Another limitation is the relative cultural homogeneity of the population. However, currently no other sources of data exist that include a large number of reports for an equally large number of substances. 
Present-day legislation, as well as ethical concerns, contribute towards the unfeasibility of obtaining such information under controlled laboratory conditions (Nutt, King, \& Nichols, 2013). While the reports in the Erowid corpus could be affected by a number of different confounds, the amount of data might be sufficiently high to expect the detection of a meaningful signal in spite of uncontrolled sources of noise (Halevy, Norvig, \& Pereira, 2009).

\section{Conclusion}

In conclusion, we have systematically compared the semantic similarity of reports associated with the use of psychoactive compounds and NDE narratives, and found evidence that ketamine (and to a lesser extent different serotonergic psychedelics and deliriant alkaloids) can produce an altered state of consciousness resembling near-death. With the available experimental data, it is neither possible to corroborate nor refute the hypothesis that the release of an endogenous ketamine-like neuroprotective agent underlies NDE phenomenology. However, our results do provide evidence that ketamine, as well as other psychoactive substances, result in a state phenomenologically similar to that of "dying" (understood as the content of NDE narratives). This could have important implications for the pharmacological induction of NDE-like states for scientific purposes, as well as for therapeutic uses in the terminally ill as means to alleviate death anxiety. We believe that the development of evidence-based treatments for such anxiety is a cornerstone of a more compassionate approach towards the universal experience of transitioning between life and death.

\section{Acknowledgments}

We acknowledge insightful discussions with David Luke, Facundo Carrillo, Mariano Sigman, Diego Fernandez Slezak, Robin Carhart-Harris, Raphaël Millière, Chris Timmermann and Hannes Kettner. We also thank the founders, curators, contributors and volunteers of Erowid Center for sharing their data and for their decades of work on the experience report collection. This study was supported by the University and University Hospital of Liege, the Belgian National Funds for Scientific Research (FRS-FNRS), the European Union's Horizon 2020 Framework Programme for Research and Innovation under the Specific Grant Agreement No. 785907 (Human Brain Project SGA2), the Luminous project (EU-H2020-fetopenga686764), the European Space Agency (ESA), Fonds Léon Frédéricq, the Bial Foundation, the Mind Science Foundation, the European Commission and the National Scientific and Technical Research Council (CONICET).

\section{Appendix A. Supplementary material}

Supplementary data to this article can be found online at https://doi.org/10.1016/j.concog.2019.01.011.

\section{References}

Addy, P. H., Garcia-Romeu, A., Metzger, M., \& Wade, J. (2015). The subjective experience of acute, experimentally-induced Salvia divinorum inebriation. Journal of Psychopharmacology, 29(4), 426-435.

Adler, C. M., Malhotra, A. K., Elman, I., Goldberg, T., Egan, M., Pickar, D., \& Breier, A. (1999). Comparison of ketamine-induced thought disorder in healthy volunteers and thought disorder in schizophrenia. The American Journal of Psychiatry, 156(10), 1646-1649.

Alberich, S., Martínez-Cengotitabengoa, M., López, P., Zorrilla, I., Núñez, N., Vieta, E., \& González-Pinto, A. (2017). Efficacy and safety of ketamine in bipolar depression: A systematic review. Revista de Psiquiatría y Salud Mental, 10(2), 104-112.

Albott, C. S., Lim, K. O., Forbes, M. K., Erbes, C., Tye, S. J., Grabowski, J. G., ... Shiroma, P. R. (2018). Efficacy, safety, and durability of repeated ketamine infusions for comorbid posttraumatic stress disorder and treatment-resistant depression. Journal of Clinical Psychiatry, 79(3).

Athappilly, G. K., Greyson, B., \& Stevenson, I. (2006). Do prevailing societal models influence reports of near-death experiences?: A comparison of accounts reported before and after 1975. The Journal of Nervous and Mental Disease, 194(3), 218-222.

Bailey, G. (1973). Changes in osage social organization. University of Oregon Anthropological Papers, 5, $1673-1906$.

Barker, S. A., Borjigin, J., Lomnicka, I., \& Strassman, R. (2013). LC/MS/MS analysis of the endogenous dimethyltryptamine hallucinogens, their precursors, and major metabolites in rat pineal gland microdialysate. Biomedical Chromatography, 27(12), 1690-1700.

Barker, S. A., McIlhenny, E. H., \& Strassman, R. (2012). A critical review of reports of endogenous psychedelic N,N-dimethyltryptamines in humans: 1955-2010. Drug testing and Analysis, 4(7-8), 617-635.

Barrett, F. S., \& Griffiths, R. R. (2018). Classic hallucinogens and mystical experiences: Phenomenology and neural correlates. Behavioral Neurobiology of Psychedelic Drugs, 36, 393-430.

Bedi, G., Cecchi, G. A., Slezak, D. F., Carrillo, F., Sigman, M., \& de Wit, H. (2014). A window into the intoxicated mind? Speech as an index of psychoactive drug effects. Neuropsychopharmacology, 39(10), 2340-2348.

Belanti, J., Perera, M., \& Jagadheesan, K. (2008). Phenomenology of near-death experiences: A cross-cultural perspective. Transcultural Psychiatry, 45(1), 121-133.

Bianco, S., Sambin, M., \& Palmieri, A. (2017). Meaning making after a near-death experience: The relevance of intrapsychic and interpersonal dynamics. Death Studies, 41(9), 562-573.

Borjigin, J., Lee, U., Liu, T., Pal, D., Huff, S., Klarr, D., ... Mashour, G. A. (2013). Surge of neurophysiological coherence and connectivity in the dying brain. Proceedings of the National Academy of Sciences of the United States of America, 110(35), 14432-14437.

Britton, W. B., \& Bootzin, R. R. (2004). Near-death experiences and the temporal lobe. Psychological Science, 15(4), 254-258.

Bubeníková-Valesová, V., Horácek, J., Vrajová, M., \& Höschl, C. (2008). Models of schizophrenia in humans and animals based on inhibition of NMDA receptors. Neuroscience \& Biobehavioral Reviews, 32(5), 1014-1023.

Carhart-Harris, R. L., Muthukumaraswamy, S., Roseman, L., Kaelen, M., Droog, W., Murphy, K., ... Nutt, D. J. (2016). Neural correlates of the LSD experience revealed by multimodal neuroimaging. Proceedings of the National Academy of Sciences of the United States of America, 113(17), 4853-4858.

Carr, D., \& Prendergast, M. (1981). Endorphins at the approach of death. The Lancet, 317.

Cassol, H., Pétré, B., Degrange, S., Martial, C., Charland-Verville, V., Lallier, F., ... Laureys, S. (2018). Qualitative thematic analysis of the phenomenology of neardeath experiences. PLoS One, 13(2), e0193001.

Charland-Verville, V., Jourdan, J. P., Thonnard, M., Ledoux, D., Donneau, A. F., Quertemont, E., \& Laureys, S. (2014). Near-death experiences in non-life-threatening events and coma of different etiologies. Frontiers in Human Neuroscience, 8, 203.

Chavkin, C., James, I. F., \& Goldstein, A. (1982). Dynorphin is a specific endogenous ligand of the kappa opioid receptor. Science, $215(4531)$, 413-415. 
Chavkin, C., Sud, S., Jin, W., Stewart, J., Zjawiony, J. K., Siebert, D. J., ... Roth, B. L. (2004). Salvinorin A, an active component of the hallucinogenic sage salvia divinorum is a highly efficacious kappa-opioid receptor agonist: Structural and functional considerations. Journal of Pharmacology and Experimental Therapeutics, 308(3), 1197-1203.

Chawla, L. S., Terek, M., Junker, C., Akst, S., Yoon, B., Brasha-Mitchell, E., \& Seneff, M. G. (2017). Characterization of end-of-life electroencephalographic surges in critically ill patients. Death Studies, 41(6), 385-392.

Church, J., Zeman, S., \& Lodge, D. (1988). The neuroprotective action of ketamine and MK-801 after transient cerebral ischemia in rats. Anesthesiology, 69(5), $702-709$.

Corazza, O., \& Schifano, F. (2010). Near-death states reported in a sample of 50 misusers. Substance Use \& Misuse, 45(6), 916-924.

Coyle, J., Presti, D., \& Baggott, M. (2012). Quantitative analysis of narrative reports of psychedelic drugs. arXiv preprint arXiv:1206.0312.

Dakwar, E., Anerella, C., Hart, C. L., Levin, F. R., Mathew, S. J., \& Nunes, E. V. (2014). Therapeutic infusions of ketamine: Do the psychoactive effects matter? Drug and Alcohol Dependence, 136, 153-157.

Dawkins, R. (2006). The selfish gene. Oxford; New York: Oxford University Press.

Devereux, P. (1997). The long trip: A prehistory of psychedelia. New York: Penguin Arkana.

Dillon, P., Copeland, J., \& Jansen, K. (2003). Patterns of use and harms associated with non-medical ketamine use. Drug and Alcohol Dependence, 69(1).

DiMaggio, D. A., Contreras, P. C., Quirion, R., \& O'Donohue, T. L. (1986). Isolation and identification of an endogenous ligand for the phencyclidine receptor. NIDA Research Monograph, 64, 24-36.

dos Santos, R. G. (2013). Safety and side effects of ayahuasca in humans-an overview focusing on developmental toxicology. Journal of Psychoactive Drugs, 45(1), $68-78$.

Dos Santos, R. G., Bouso, J. C., Alcázar-Córcoles, M., \& Hallak, J. E. C. (2018). Efficacy, tolerability, and safety of serotonergic psychedelics for the management of mood, anxiety, and substance-use disorders: A systematic review of systematic reviews. Expert Review of Clinical Pharmacology, 11(9), 889-902.

Dougherty, C. M. (1990). The near-death experience as a major life transition. Holistic Nursing Practice, 4(3), 84-90.

Duffy, N., \& Olson, M. (2007). Supporting a patient after a near-death experience. Nursing, 37(4), 46-48.

El-Dabbas, S. W., \& Evans, W. C. (1982). Alkaloids of the genus datura, section brugmansia. Planta Medica, 44(3), $184-185$.

Escohotado, A. (1999). A brief history of drugs: From the Stone Age to the stoned age. Rochester, Vt: Park Street Press.

Facco, E., \& Agrillo, C. (2012). Near-death-like experiences without life-threatening conditions or brain disorders: A hypothesis from a case report. Frontiers in Psychology, 3, 490.

Fenwick, P. (1997). Is the near-death experience only N-methyl-D-aspartate blocking? Journal of Near-Death Studies, 16, 42-53.

Forstmann, M., \& Sagioglou, C. (2017). Lifetime experience with (classic) psychedelics predicts pro-environmental behavior through an increase in nature relatedness. Journal of Psychopharmacology, 31(8), 975-988.

Frecska, E., Szabo, A., Winkelman, M. J., Luna, L. E., \& McKenna, D. J. (2013). A possibly sigma-1 receptor mediated role of dimethyltryptamine in tissue protection, regeneration, and immunity. J Neural Transm (Vienna), 120(9), 1295-1303.

French, C. C. (2005). Near-death experiences in cardiac arrest survivors. Progress in Brain Research, 150, $351-367$.

Fujikawa, D. G. (1995). Neuroprotective effect of ketamine administered after status epilepticus onset. Epilepsia, 36(2), $186-195$.

Gasser, P., Holstein, D., Michel, Y., Doblin, R., Yazar-Klosinski, B., Passie, T., \& Brenneisen, R. (2014). Safety and efficacy of lysergic acid diethylamide-assisted psychotherapy for anxiety associated with life-threatening diseases. The Journal of Nervous and Mental Disease, 202(7), 513-520.

Gasser, P., Kirchner, K., \& Passie, T. (2015). LSD-assisted psychotherapy for anxiety associated with a life-threatening disease: A qualitative study of acute and sustained subjective effects. Journal of Psychopharmacology, 29(1), 57-68.

Glue, P., Neehoff, S. M., Medlicott, N. J., Gray, A., Kibby, G., \& McNaughton, N. (2018). Safety and efficacy of maintenance ketamine treatment in patients with treatment-refractory generalised anxiety and social anxiety disorders. Journal of Psychopharmacology, 32(6), 663-667.

Greyson, B. (1983). The near-death experience scale. Construction, reliability, and validity. The Journal of Nervous and Mental Disease, 171(6), 369-375.

Greyson, B. (1993). Varieties of near-death experience. Psychiatry, 56(4), 390-399.

Greyson, B. (1997). The near-death experience as a focus of clinical attention. The Journal of Nervous and Mental Disease, 185(5), 327-334.

Greyson, B. (2001). Posttraumatic stress symptoms following near-death experiences. American Journal of Orthopsychiatry, 71(3), $368-373$.

Greyson, B. (2006). Near-death experiences and spirituality. Journal of Religion and Science, 41(2), 393-414.

Greyson, B. (2007b). Reduced death threat in near-death experiencers. Death Studies, 16(6), 523-536.

Greyson, B. (2007a). Consistency of near-death experience accounts over two decades: Are reports embellished over time? Resuscitation, 73(3), 407-411.

Greyson, B. (2008). The near-death experience. Alternative Therapies in Health and Medicine, 14(3), 14 author reply 14-15.

Greyson, B., \& Bush, N. E. (1992). Distressing near-death experiences. Psychiatry, 55(1), 95-110.

Greyson, B., Holden, J. M., \& van Lommel, P. (2012). There is nothing paranormal about near-death experiences' revisited: Comment on Mobbs and Watt. Trends in Cognitive Sciences, 16.

Greyson, B., Kelly, E. F., \& Dunseath, W. J. (2013). Surge of neurophysiological activity in the dying brain. Proceedings of the National Academy of Sciences of the United States of America, 110(47), E4405.

Griffiths, R. R., Johnson, M. W., Carducci, M. A., Umbricht, A., Richards, W. A., Richards, B. D., ... Klinedinst, M. A. (2016). Psilocybin produces substantial and sustained decreases in depression and anxiety in patients with life-threatening cancer: A randomized double-blind trial. Journal of Psychopharmacology, 30(12), 1181-1197.

Grinspoon, L., \& Bakalar, J. B. (1979). Psychedelic drugs reconsidered. New York: Basic Books.

Grob, C. S., Danforth, A. L., Chopra, G. S., Hagerty, M., McKay, C. R., Halberstadt, A. L., \& Greer, G. R. (2011). Pilot study of psilocybin treatment for anxiety in patients with advanced-stage cancer. Archives of General Psychiatry, 68(1), 71-78.

Grof, S., \& Halifax, J. (1977). The human encounter with death. New York: E. P. Dutton.

Hähnke, V. D., Kim, S., \& Bolton, E. E. (2018). PubChem chemical structure standardization. Journal of Cheminformatics, $10(1), 36$.

Halevy, A., Norvig, P., \& Pereira, F. (2009). The unreasonable effectiveness of data. IEEE Intelligent Systems, 24(2), 8-12.

Holaday, J. W., \& Faden, A. I. (1978). Naloxone reversal of endotoxin hypotension suggests role of endorphins in shock. Nature, $275(5679), 450-451$.

Huang, T. S., \& Narendra, P. M. (1975). Image restoration by singular value decomposition. Applied Optics, 14(9), $2213-2216$.

Hudetz, J. A., \& Pagel, P. S. (2010). Neuroprotection by ketamine: A review of the experimental and clinical evidence. Journal of Cardiothoracic and Vascular Anesthesia, 24(1), 131-142.

Iglesias-Lepine, M. L., Mariñosa, M., Ferrer-Da Pena, M. D., \& Epelde, F. (2012). The revival of datura and brugmansia as recreational drugs. Medicina clinica, 139(2), 90-91.

Jansen, K. (1989). Near death experience and the NMDA receptor. BMJ, 298(6689), 1708.

Jansen, K. L. (1990). Neuroscience and the near-death experience: Roles for the NMSA-PCP receptor, the sigma receptor and the endopsychosins. Medical Hypotheses, 31(1), 25-29.

Jansen, K. (1997b). The ketamine model of the near-death experience: A central role for the N-methyl-D-aspartate receptor. Journal of Near - Death Studies, 16(1), $5-26$.

Jansen, K. (1997a). Response to commentaries on the ketamine model of the near-death experience. Journal of Near-Death Studies, 16(1), 79-95.

Katz, J., Saadon-Grosman, N., \& Arzy, S. (2017). The life review experience: Qualitative and quantitative characteristics. Consciousness and Cognition, 48, 76-86.

Kellehear, A. (1990). The near-death experience as status passage. Social Science and Medicine, 31(8), 933-939.

Kellehear, A. (1993). Culture, biology, and the near-death experience: A reappraisal. Journal of Nervous and Mental Disease, 181(3), 148-156.

Kelly, E. W. (2001). Near-death experiences with reports of meeting deceased people. Death Studies, 25(3), 229-249.

Kolp, E. (2007). Ketamine-enhanced psychotherapy: Preliminary clinical observations on its effects in treating death anxiety. International Journal of Transpersonal Studies, 26(1). 
Kraehenmann, R. (2017). Dreams and psychedelics: Neurophenomenological comparison and therapeutic implications. Current Neuropharmacology, 15(7), 1032-1042. Kyzar, E. J., Nichols, C. D., Gainetdinov, R. R., Nichols, D. E., \& Kalueff, A. V. (2017). Psychedelic Drugs in Biomedicine. Trends in Pharmacological Sciences, 38(11), 992-1005.

Landauer, T. K., Laham, D., \& Derr, M. (2004). From paragraph to graph: Latent semantic analysis for information visualization. Proceedings of the National Academy of Sciences of the United States of America, 101(Suppl. 1), 5214-5219.

Lange, R., Greyson, B., \& Houran, J. (2004). A Rasch scaling validation of a 'core'near-death experience. British Journal of Psychology, 95(2), $161-177$.

Lange, R., Greyson, B., \& Houran, J. (2015). Using computational linguistics to understand near-death experiences: Concurrent validity for the Near Death Experience Scale. Psychology of Consciousness: Theory, Research, and Practice, 2(1).

Laureys, S., Gosseries, O., \& Tononi, G. (2016). The neurology of consciousness: Cognitive neuroscience and neuropathology. Amsterdam; Boston: Elsevier/AP, Academic Press is an imprint of Elsevier.

Lazarewicz, M. T., Ehrlichman, R. S., Maxwell, C. R., Gandal, M. J., Finkel, L. H., \& Siegel, S. J. (2010). Ketamine modulates theta and gamma oscillations. Journal of Cognitive Neuroscience, 22(7), 1452-1464.

Leary, T., Metzner, R., Ram, D., \& Karma glin, P. (1966). The psychedelic experience. n.p., Folkways Records FL 9701. c1966.

Lee, M. A., \& Shlain, B. (1992). Acid dreams: The complete social history of LSD: The CIA, the sixties, and beyond. New York: Grove Weidenfeld.

Lewin, L., \& Wirth, P. H. A. (1931). Phantastica, narcotic and stimulating drugs; their use and abuse. New York: E.P. Dutton \& Company.

Loh, H. H., Tseng, L. F., Wei, E., \& Li, C. H. (1976). beta-endorphin is a potent analgesic agent. Proceedings of the National Academy of Sciences of the United States of America, 73(8), 2895-2898.

Luke, D. (2008). Psychoactive substances and paranormal phenomena: A comprehensive review. International Journal of Transpersonal Studies, $31(1)$, 12.

Luna, L. (2011). Indigenous and mestizo use of ayahuasca. The Ethnopharmacology of Ayahuasca, 1(21).

Lyons, T., \& Carhart-Harris, R. L. (2018). Increased nature relatedness and decreased authoritarian political views after psilocybin for treatment-resistant depression. Journal of Psychopharmacology, 32(7), 811-819.

Maas, U., \& Süster, S. (2003). Music in the Iboga-initiation ceremony in Gabon: Polyrhythms supporting a pharmacotherapy. Music Therapy Today, 5(3).

Marcoux, F. W., Goodrich, J. E., \& Dominick, M. A. (1988). Ketamine prevents ischemic neuronal injury. Brain Research, 452(1-2), 329-335.

Martial, C., Cassol, H., Antonopoulos, G., Charlier, T., Heros, J., Donneau, A. F., ... Laureys, S. (2017). Temporality of features in near-death experience narratives. Frontiers in Human Neuroscience, 11, 311.

McKetin, R. (2018). Methamphetamine psychosis: Insights from the past. Addiction, 113(8), 1522-1527.

Meduna, L. (1950). The effect of carbon dioxide upon the functions of the brain. Springfield (IL): Charles.

Millière, R., Carhart-Harris, R., Roseman, L., Trautwein, F.-M., \& Berkovich-Ohana, A. (2018). Psychedelics, meditation, and self-consciousness. Frontiers in Psychology, 9(1475).

Minarro-Giménez, J. A., Marín-Alonso, O., \& Samwald, M. (2014). Exploring the application of deep learning techniques on medical text corpora. Studies in Health Technology and Informatics, 205, 584-588.

Mobs, D., \& Watt, C. (2011). There is nothing paranormal about near-death experiences: How neuroscience can explain seeing bright lights, meeting the dead, or being convinced you are one of them. Trends in Cognitive Sciences, 15(10), 447-449.

Moore, L. E., \& Greyson, B. (2017). Characteristics of memories for near-death experiences. Consciousness and Cognition, 51, $116-124$.

Moody, Raymond A. (1975). Life After Life: The Investigation of a Phenomena, Survival of Bodily Death. Mockingbird Books.

Morse, M. L., Venecia, D., \& Milstein, J. (1989). Near-death experiences: A neurophysiologic explanatory model. Journal of Near-death studies, 8(1), 45-53.

Muthukumaraswamy, S. D. (2013). High-frequency brain activity and muscle artifacts in MEG/EEG: A review and recommendations. Frontiers in Human Neuroscience, 7, 1-11. https://doi.org/10.3389/fnhum.2013.00138.

Muthukumaraswamy, S. D., Carhart-Harris, R. L., Moran, R. J., Brookes, M. J., Williams, T. M., Errtizoe, D., ... Nutt, D. J. (2013). Broadband cortical desynchronization underlies the human psychedelic state. Journal of Neuroscience, 33(38), 15171-15183.

Muthukumaraswamy, S. D., \& Liley, D. T. (2018). 1/f electrophysiological spectra in resting and drug-induced states can be explained by the dynamics of multiple oscillatory relaxation processes. NeuroImage, 179, 582-595.

Muthukumaraswamy, S. D., Shaw, A. D., Jackson, L. E., Hall, J., Moran, R., \& Saxena, N. (2015). Evidence that subanesthetic doses of ketamine cause sustained disruptions of NMDA and AMPA-mediated frontoparietal connectivity in humans. Journal of Neuroscience, 35(33), 11694-11706.

Nichols, D. E. (1986). Differences between the mechanism of action of MDMA, MBDB, and the classic hallucinogens. Identification of a new therapeutic class: Entactogens. Journal of Psychoactive Drugs, 18(4), 305-313.

Nichols, D. E. (2016). Psychedelics. Pharmacological Reviews, 68(2), 264-355.

Nichols, D. E. (2018). N, N-dimethyltryptamine and the pineal gland: Separating fact from myth. Journal of Psychopharmacology, 32(1), 30-36.

Niciu, M. J., Shovestul, B. J., Jaso, B. A., Farmer, C., Luckenbaugh, D. A., Brutsche, N. E., ... Zarate, C. A. (2018). Features of dissociation differentially predict antidepressant response to ketamine in treatment-resistant depression. Journal of Affective Disorders, 232, 310-315.

Noyes, R. (1980). Attitude change following near-death experiences. Psychiatry, 43(3), 234-242.

Nutt, D. J., King, L. A., \& Nichols, D. E. (2013). Effects of Schedule I drug laws on neuroscience research and treatment innovation. Nature Reviews Neuroscience, 14(8), 577-585.

Owens, J. E., Cook, E. W., \& Stevenson, I. (1990). Features of "near-death experience" in relation to whether or not patients were near death. Lancet, 336(8724), $1175-1177$.

Palhano-Fontes, F., Barreto, D., Onias, H., Andrade, K. C., Novaes, M. M., Pessoa, J. A., Mota-Rolim, S. A., Osório, F. L., Sanches, R., Dos Santos, R. G., Tófoli, L. F., de Oliveira Silveira, G., Yonamine, M., Riba, J., Santos, F. R., Silva-Junior, A. A., Alchieri, J. C., Galvão-Coelho, N. L., Lobão-Soares, B., Hallak, J. E. C., Arcoverde, E., Maia-de-Oliveira, J. P., \& Araújo, D. B. (2018). Rapid antidepressant effects of the psychedelic ayahuasca in treatment-resistant depression: A randomized placebocontrolled trial. Psychological Medicine, 1-9.

Parnia, S., Spearpoint, K., \& Fenwick, P. B. (2007). Near death experiences, cognitive function and psychological outcomes of surviving cardiac arrest. Resuscitation, $74(2), 215-221$.

Perrine, D. M. (1996). The chemistry of mind-altering drugs : History, pharmacology, and cultural context. Washington, DC: American Chemical Society.

Rabi, J. (2016). Topical ketamtene: A review of the history, mechanisms, uses, safety, and future. International Journal of Pharmaceutical Compounding, 20(2), 107-113.

Rätsch, C. (2005). The encyclopedia of psychoactive plants: Ethnopharmacology and its applications. Rochester, Vt: Park Street Press.

Ring, K. (1982). Life at death: A scientific investigation of the near-death experience. New York: Quill.

Ring, K. (1988). Guest editorial: Paradise is paradise: Reflections on psychedelic drugs, mystical experience, and the near-death experience. Journal of Near-Death Studies, 6(3), 138-148.

Riva-Posse, P., Reiff, C. M., Edwards, J. A., Job, G. P., Galendez, G. C., Garlow, S. J., ... McDonald, W. M. (2018). Blood pressure safety of subanesthetic ketamine for depression: A report on 684 infusions. Journal of Affective Disorders, 236, 291-297.

Roth, B. L., Baner, K., Westkaemper, R., Siebert, D., Rice, K. C., Steinberg, S., ... Rothman, R. B. (2002). Salvinorin A: A potent naturally occurring nonnitrogenous kappa opioid selective agonist. Proceedings of the National Academy of Sciences of the United States of America, 99(18), 11934-11939.

Rothman, S. M., \& Olney, J. W. (1986). Glutamate and the pathophysiology of hypoxic-ischemic brain damage. Annals of Neurology, $19(2), 105-111$.

Royse, D., \& Badger, K. (2017). Near-death experiences, posttraumatic growth, and life satisfaction among burn survivors. Social Work in Health Care, 56(3), 155-168.

Saavedra-Aguilar, J. C., \& Gómez-Jeria, J. S. (1989). A neurobiological model for near-death experiences. Journal of Near-Death Studies, 7(4), $205-222$.

Sanz, C., Zamberlan, F., Erowid, E., Erowid, F., \& Tagliazucchi, E. (2018). The experience elicited by hallucinogens presents the highest similarity to dreaming within a large database of psychoactive substance reports. Frontiers in Neuroscience, 12, 7.

Sattar, Y., Wilson, J., Khan, A. M., Adnan, M., Azzopardi Larios, D., Shrestha, S., Rahman, Q., Mansuri, Z., Hassan, A., Patel, N. B., Tariq, N., Latchana, S., Lopez Pantoja, S. C., Vargas, S., Shaikh, N. A., Syed, F., Mittal, D., \& Rumesa, F. (2018). A review of the mechanism of antagonism of N-methyl-D-aspartate receptor by ketamine in treatment-resistant depression. Cureus, 10(5). 
Schenberg, E. E., Alexandre, J. F. M., Filev, R., Cravo, A. M., Sato, J. R., Muthukumaraswamy, S. D., Yonamine, M., Waguespack, M., Lomnicka, I., Barker, S., \& da Silveira, D. X. (2015). Acute biphasic effects of ayahuasca. PLoS One, 10(9).

Schultes, R. E., Hofmann, A., \& Rätsch, C. (2001). Plants of the gods: their sacred, healing, and hallucinogenic powers. Rochester, Vt.: Healing Arts Press.

Schultes, R. E., \& Raffauf, R. F. (2004). Vine of the soul: Medicine men, their plants and rituals in the Colombian Amazonia. Sante Fe, N.M.: Synergetic Press.

Schwaninger, J., Eisenberg, P. R., Schechtman, K. B., \& Weiss, A. N. (2002). A prospective analysis of near-death experiences in cardiac arrest patients. Journal of NearDeath Studies, 20(4), 215-232.

Shapiro, H. (1988). Waiting for the man: The story of drugs and popular music. New York: Morrow.

Sheffler, D. J., \& Roth, B. L. (2003). Salvinorin A: The "magic mint" hallucinogen finds a molecular target in the kappa opioid receptor. Trends in Pharmacological Sciences, 24(3), 107-109.

Shulgin, A. T., \& Shulgin, A. (1997). TIHKAL: the continuation. Berkeley, Calif., Transform. .

Siegel, R. (1984). Hashish near-death experiences. The Journal of Near-Death Studies, 4, 70-86.

Sivanesan, E., Gitlin, M. C., \& Candiotti, K. A. (2017). Opioid-induced hallucinations: A review of the literature, pathophysiology, diagnosis, and treatment. Survey of Anesthesiology, 61(1), 21.

Sleutjes, A., Moreira-Almeida, A., \& Greyson, B. (2014). Almost 40 years investigating near-death experiences: An overview of mainstream scientific journals. The Journal of Nervous and Mental Disease, 202(11), 833-836.

Sotelo, J., Perez, R., Guevara, P., \& Fernandez, A. (1995). Changes in brain, plasma and cerebrospinal fluid contents of beta-endorphin in dogs at the moment of death. Neurological Research, 17(3), 223-225.

Strassman, R. (1997). Endogenous ketamine-like compounds and the NDE: If so, so what? Journal of Near-Death Experiences, 16.

Strassman, R. (2001). DMT: The spirit molecule : A doctor's revolutionary research into the biology of near-death and mystical experiences. Rochester, Vt: Park Street Press.

Strassman, R. J., Qualls, C. R., Uhlenhuth, E. H., \& Kellner, R. (1994). Dose-response study of N, N-dimethyltryptamine in humans. II. Subjective effects and preliminary results of a new rating scale. Archives of General Psychiatry, 51(2), 98-108.

Studerus, E., Gamma, A., Kometer, M., \& Vollenweider, F. X. (2012). Prediction of psilocybin response in healthy volunteers. PLoS ONE, 7(2), e30800.

Sumnall, H. R., Measham, F., Brandt, S. D., \& Cole, J. C. (2011). Salvia divinorum use and phenomenology: Results from an online survey. Journal of Psychopharmacology, 25(11), 1496-1507.

Szabo, A., Kovacs, A., Riba, J., Djurovic, S., Rajnavolgyi, E., \& Frecska, E. (2016). The endogenous hallucinogen and trace amine N,N-dimethyltryptamine (DMT) displays potent protective effects against hypoxia via sigma-1 receptor activation in human primary iPSC-derived cortical neurons and microglia-like immune cells. Frontiers in Neuroscience, 10, 423.

Timmermann, C., Roseman, L., Williams, L., Erritzoe, D., Martial, C., Cassol, H., ... Carhart-Harris, R. (2018). DMT models the near-death experience. Frontiers in Psychology, 9, 1424.

Tognoli, E., \& Kelso, J. A. (2014). Enlarging the scope: Grasping brain complexity. Frontiers in Systems Neuroscience, 8, 122.

Tononi, G. (2012). Integrated information theory of consciousness: An updated account. Archives Italiennes de Biologie, 150(4), $293-329$.

Tononi, G., \& Edelman, G. M. (1998). Consciousness and complexity. Science, 282(5395), 1846-1851.

Toro, G., \& Thomas, B. (2007). Drugs of the dreaming : Oneirogens: Salvia divinorum and other dream-enhancing plants. Rochester, Vt: Park Street Press.

Vohra, R., Seefeld, A., Cantrell, F. L., \& Clark, R. F. (2011). Salvia divinorum: Exposures reported to a statewide poison control system over 10 years. Journal of Emergency Medicine, 40(6), 643-650.

Wallach, J., \& Brandt, S. D. (2018). Phencyclidine-based new psychoactive substances. Handbook of Experimental Pharmacology.

Wilkins, L. K., Girard, T. A., \& Cheyne, J. A. (2011). Ketamine as a primary predictor of out-of-body experiences associated with multiple substance use. Consciousness and Cognition, 20(3), 943-950.

Winstock, A. R., Kaar, S., \& Borschmann, R. (2014). Dimethyltryptamine (DMT): Prevalence, user characteristics and abuse liability in a large global sample. Journal of Psychopharmacology, 28(1), 49-54. 